\title{
Local Parametric Approach of Wireless Capsule Endoscope Localization Using Randomly Scattered Path Loss Based WCL
}

\author{
Umma Hany and Lutfa Akter \\ Department of Electrical and Electronic Engineering, Bangladesh University of Engineering and Technology, Dhaka 1205, Bangladesh \\ Correspondence should be addressed to Umma Hany; ummahany@gmail.com
}

Received 6 July 2017; Accepted 24 October 2017; Published 4 December 2017

Academic Editor: Paolo Barsocchi

Copyright (C) 2017 Umma Hany and Lutfa Akter. This is an open access article distributed under the Creative Commons Attribution License, which permits unrestricted use, distribution, and reproduction in any medium, provided the original work is properly cited.

\begin{abstract}
We propose scattered path loss based weighted centroid localization (WCL) algorithm for wireless video capsule endoscope (VCE). The main challenge in this approach is the random deviation in the measured received signal strength indicator (RSSI) caused by multipath propagation and shadowing effects of human body channel which in turn increases the localization error. To address this issue, we propose local parameter dependent path loss representation in the training phase and apply adaptive least square error (LSE) method to extract the parameters. Then, in the test phase, we estimate distance using the extracted parameters and the randomly scattered path loss. The position of capsule is estimated using non-degree based WCL followed by a calibration process. We propose suboptimal method of estimating the calibration coefficient and also compute the optimal value of coefficient analytically to set the benchmark. We develop a simulation platform using MATLAB to present the results and to verify the performance. We gradually increase the number of sensors and place them in different topologies using different dimensions. The obtained accuracy by our proposed suboptimal method of WCL is very close to the optimal benchmark for all cases. Our proposed approach also outperforms existing works in terms of localization accuracy.
\end{abstract}

\section{Introduction}

Wireless video capsule endoscopes (VCEs) are used to diagnose lesions along digestive tracts. For proper diagnosis, it is necessary to know the exact location of the lesions. VCE localization is a process of determining the unknown location of the capsule while it travels through the gastrointestinal (GI) tract. A group of sensors are used to localize the capsule. There are several positioning techniques in the literature [1], in general classified into range based [2-4] and range-free $[5,6]$. Trilateration $[2,3]$ is a simple positioning technique, where the position of the mobile target is estimated by intersection of the circles centered on the fixed anchor node's position, with a radius equal to the estimated distance between the mobile node and the anchor node. Triangulation $[3,4]$ uses the properties of triangles to calculate distances for position information. Centroid localization (CL) [5] is a range-free algorithm which localizes the target to the centroid of a set of reference points. Weighted centroid localization (WCL) [6] gives more weights to nearer reference node than the remote reference nodes.
The reported VCE localization algorithms in the literature [7] are based on either magnetic field or electric field strength. The method in [8] is based on magnetic field strength which reported $1.8 \mathrm{~mm}$ average localization error. Though the accuracy of [8] is high, the method requires more space in the capsule. Since VCE is equipped with a radio communication system, developing localization system using RF techniques got attention. In [9], the authors propose position estimation based on RFID (Radio Frequency Identification) and the 3D antenna array. In [9], the RFID of antennas which detect the tag are measured and then 3D coordinates of those antennas are used to estimate location applying the center of gravity location estimation algorithm. Their system does not require RF signal attenuation model as they do not need to measure RSS. They report mean estimation error of $2 \mathrm{~cm}$ with dense antenna arrangement. In [10], the authors propose linear approximation techniques on the data received from RSSI to predict the initial position of the object using linear least square (LLS) based trilateration and based on the initial prediction, the final position of the object is computed using nonlinear least square iteration method. In [10], the exact 
distances between the object and the sensors and simultaneous RSSI measurements from several access points (AP) are needed for accurate estimation of position. For most of the positions, an average error of $25 \%$ is reported in [10]. In $[11,12]$, the authors use RSS triangulation and Monte Carlo simulation for multicapsule cooperative localization in the GI tract and conclude that better accuracy is possible to be achieved by increasing the number of receiver sensors. They [11] achieve average localization error of $50 \mathrm{~mm}$ in the digestive organs for capsule endoscopy using more than 32 sensors on body surface and in [12] average localization error of $22.4 \mathrm{~mm}$ is achieved using 64 sensors on body surface. In [13], the authors model the medical implant communication service (MICS) band signals using finite difference time-domain (FDTD) simulation with a numerical human model and propose maximum likelihood (ML) based position estimation using RSSI. They reported [13] $38 \mathrm{~mm}$ root mean square error (RMSE) if the channel parameters are accurately measured whereas the RMSE may reach as high as $75 \mathrm{~mm}$ if the channel parameters are not accurately estimated. However, the method in [13] cannot give an accurate estimate of the capsule endoscope location with inaccurate estimated channel parameters. The authors in [14] develop a time of arrival (TOA) and path loss based one-stage method using convex optimization and spatial sparsity in 3D space considering massive multipath and shadow fading conditions. To achieve better accuracy, their proposed system must have prior and accurate knowledge on the location of grid points and the average velocity of propagation which depends on relative permittivity of the body tissues at each grid point. As per their report [14], the method can achieve approximately $1.5 \mathrm{~cm}$ RMSE with standard deviation $1.5 \mathrm{~cm}$ using 16 sensors. In [15], the authors use LLS of estimated distances and the coordinates to find initial coordinates and then nonlinear least square method is applied to find the final positions. The position estimation error reported in [15] is within $1 \mathrm{~cm}$ for $2 \mathrm{D}$ (for both homogeneous and heterogeneous phantom) and within $1 \mathrm{~cm}$ for $3 \mathrm{D}$ case for homogeneous cylinder case only. However, here the authors use the homogeneous electrical properties of muscle tissue on the simulated data from the heterogeneous phantom. For practical scenario, proper electrical properties of the nonhomogeneous tissues should be estimated and used. In [16], the nonlinear direction-of-arrival (DOA) and inertial measurement unit (IMU) measurements are integrated to track the movement of capsule using unscented Kalman filter (UKF). However, the computational complexity of their [16] proposed method is high. In [17], the authors propose hybrid method using camera motion tracking algorithm and RF localization to localize the capsule and achieve low localization error of $23 \mathrm{~mm}$ on average. In [18], the authors propose a simple localization method using WCL and apply a calibration method using LLS of estimated and real positions. However, the method in [18] is not suitable for practical scenario as it requires prior knowledge of real positions for the calibration process. The authors in [18] report mean localization error of $5.03 \mathrm{~mm}$ using eight sensors. In [19], the authors propose suboptimal degree based WCL to estimate position of capsule and report $6.27 \mathrm{~mm}$ RMSE error. In [18, 19], the path loss is linearized assuming minimum path loss deviation which is not realistic for human body environment. The authors in [20] propose Gaussian weighted average (GWA) based and MIMO based nonparametric methods of path loss estimation using UWB channel. The authors in [20] also propose calibrated WCL (CWCL) for VCE localization and report $5.14 \mathrm{~mm}$ RMSE error using MIMO based CWCL.

A major challenge in path loss based VCE localization algorithms is the modeling of human body channel which suffers severe multipath propagation and heavy shadow fading effects caused by the internal organs and nonhomogeneous medium of propagation $[13,21,22]$. The random variation in RSSI measurements and path loss estimations result in large localization error [22]. Thus, the randomness issue of channel needs to be addressed to improve the localization accuracy of VCE.

Trilateration and triangulation based VCE localization algorithms [10-12] compute the location using distance and angle information of triangle formed by three reference sensor nodes [23]. For more number of sensors, the algorithms in [10-12] require several combinations of three sensor nodes to compute the location [23] which may increase computational complexity of the localization. The performance of the algorithms in [10-12] also decreases excessively with estimation errors in channel [23]. The other methods reported in the literature [10-15] also require very precise knowledge on channel parameters or relative permittivity of the body tissues for accurate estimation of positions. As human body is a complex environment of experimentation, a simple localization approach with less computational complexity is required. WCL [6] is a localization approach [12] which can estimate location using three or more sensors with less computational complexity, less hardware cost, and less communication overhead $[12,24]$. It is simple as well as robust to errors in the estimated channel model parameters $[12,23]$. It has attracted a lot of interest [25-31] in outdoor environment. Though it is dependent on beacon numbers and placements, the accuracy can be significantly improved using more number of sensors [23]. Thus for a complex nonhomogeneous environment as human body, WCL is an appropriate choice for localization of VCE.

In this paper, we propose a simple localization approach for VCE using scattered path loss based WCL in presence of vast multipath propagation and shadow fading conditions of nonhomogeneous human body channel. To address the randomness issue in the measured path loss, we propose local parameter dependent path loss representation. In the training phase, we divide the traveling path of capsule into clusters of neighboring points and propose adaptive least square error (LSE) method to find local path loss parameters of the clusters. In the test phase, the local parameters and the measured path loss (randomly scattered) are used to calculate the distance of the sensors from the target. Then we calculate the weight of the sensors position using the calculated distances. Finally, the position of the capsule is estimated using WCL by finding weighted average of the sensors position. We propose suboptimal method of computing calibration coefficient to improve the accuracy of localization. We also analytically compute the optimal value of coefficient to set the 


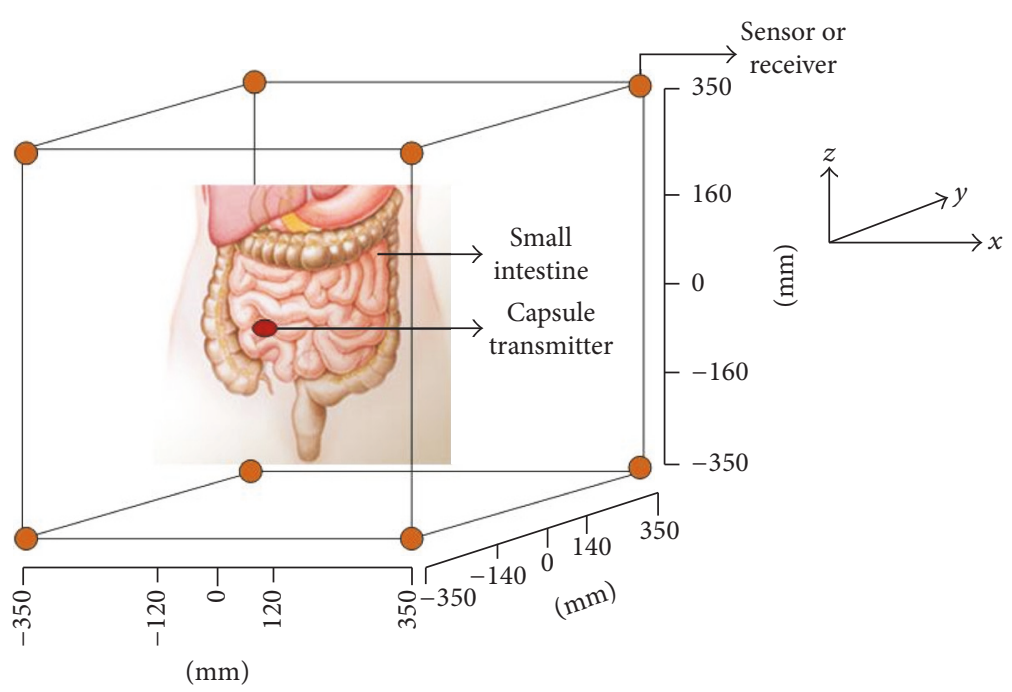

FIgURE 1: Video capsule endoscope (VCE) localization scenario.

benchmark of accuracy. The optimal coefficient is calculated using real positions of capsule which is not practically applicable and used only to set the benchmark of accuracy for comparison purpose. The suboptimal method calculates the coefficient using maximum distance of target and sensors which is realistic for practical implementation. We develop a simulation platform using MATLAB to show the results and to verify the accuracy of our proposed algorithm using different performance indices. We verify the accuracy by gradually increasing the number of sensors and place them following different topologies in different dimensions. We observe improved accuracy in all cases. We observe that using optimal coefficient based method, root mean square error (RMSE) of $13.17 \mathrm{~mm}$ is obtained whereas using our proposed suboptimal method, RMSE of $10.43 \mathrm{~mm}$ is achievable without prior knowledge of real positions which is very close to the benchmark accuracy.

\section{System Overview}

We consider a 3D sensor array to localize the video endoscope capsule (VCE) equipped with a radio transmitter (Tx) as shown in Figure 1. The capsule transmitter uses a MICS band multiturn loop antenna to emit radio wave while it travels through the small intestine. The sensors are configured as RF receivers $(\mathrm{Rx})$ which receive radio signals transmitted from the capsule transmitter. The cubical sensor array consists of a number of sensors (8-64) placed at the corner points which may be set on a body surrounded belt or jacket. The receivers measure the received signal strength indicator (RSSI) of signal transmitted from the VCE traveling through $M$ possible coordinate points inside the small intestine of $280 \mathrm{~mm} \times 120 \mathrm{~mm} \times 320 \mathrm{~mm}$ dimension. The measured RSSI is sent to the central unit for further processing. The central unit is incorporated with the localization tool which is used to estimate the $3 \mathrm{D}$ position of the capsule using our proposed algorithms. Most of the notations and symbols used in the whole paper are listed in Notations.

\section{Path Loss Model}

The radio propagation characteristics change with distance, medium, and environment. The radio propagation channel is modeled by analyzing the signal propagation from $\mathrm{Tx}$ to $\mathrm{Rx}$ for a number of experimental locations. The received power by an antenna at distance $d$ from the radiating transmitter is given by Friis equation [32] as

$$
\text { Received Power: } P_{r}(d)=\frac{P_{t} G_{t} G_{r} \lambda^{2}}{(4 \pi)^{2} d^{2} L}
$$

where $P_{t}$ is the transmitted power, $P_{r}(d)$ is the received power which is a function of the Tx-Rx separation distance $d, G_{t}$ is the transmitter antenna gain, $G_{r}$ is the received power gain, $L$ is the system loss factor not related to propagation $(L \geq 1)$, and $\lambda$ is the wavelength. An isotropic antenna radiates power in all directions uniformly with unit gain. The path loss $(S)$ is the signal attenuation measured in $\mathrm{dB}$ which is represented as the difference between the transmitted $\left(P_{T}\right)$ and the received power (RSSI) as a positive quantity [32] as

$$
\begin{aligned}
S(\mathrm{~dB}) & =P_{T}(\mathrm{dBm})-\operatorname{RSSI}(\mathrm{dBm}) \\
& =10 \log \left(\frac{P_{t}}{P_{r}}\right) .
\end{aligned}
$$

As we have assumed that the VCE travels through $M$ possible coordinate points inside the small intestine, thus each of the sensors receives signals from $M$ possible positions of the VCE. The sensors measure the RSSI and then the path loss is calculated from measured RSSI using (2). The propagation model based on theory and measurement shows that average 
received signal strength diminishes logarithmically with separation distance. The log-distance path loss model [32] can be used to model the average path loss for a range of Tx-Rx separation distance $d(i, j)$ as

$$
\overline{S(i, j)}=\operatorname{PL}\left(d_{0}\right)+10 \alpha \log \left(\frac{d(i, j)}{d_{0}}\right),
$$

where $\alpha$ is the path loss exponent which is dependent on environment; $d_{0}$ is the reference distance which is less than $d(i, j)$. However, (4) does not consider all the environmental factors. In VCE localization system, the transmitter propagates through the human body which is a nonhomogeneous environment. Thus, the path loss is deviated due to the random shadowing effects caused by the nonhomogeneous environment which leads to vastly different measured signal at two different locations having the same Tx-Rx separation distance. The path loss $S(i, j)$ of the signal from $i$ th position of the target to the $j$ th sensor through human body is random and log-normally distributed (normal in $\mathrm{dB}$ ) around a mean value which can be expressed using the log-normal shadowing model [32] as

$$
\begin{aligned}
S(i, j) & =\overline{S(i, j)}+X_{\sigma} \\
& =\operatorname{PL}\left(d_{0}\right)+10 \alpha \log _{10}\left(\frac{d(i, j)}{d_{0}}\right)+X_{\sigma},
\end{aligned}
$$

where $d(i, j)$ is the distance of the $i$ th position of target from the $j$ th sensor and $X_{\sigma}$ is a zero-mean Gaussian random variable (in $\mathrm{dB}$ ) with standard deviation $\sigma$ (in $\mathrm{dB}$ ). The random scattered path loss of $M$ possible target positions from any sensor $j$ is given as

$$
\begin{aligned}
& \left(\begin{array}{c}
S(1, j) \\
S(2, j) \\
\vdots \\
S(M, j)
\end{array}\right) \\
& =\left(\begin{array}{ccc}
1 & 10 \log _{10}\left(\frac{d(1, j)}{d_{0}}\right) & 1 \\
1 & 10 \log _{10}\left(\frac{d(2, j)}{d_{0}}\right) & 1 \\
\vdots & \vdots & \vdots \\
1 & 10 \log _{10}\left(\frac{d(M, j)}{d_{0}}\right) & 1
\end{array}\right)\left(\begin{array}{c}
\mathrm{PL}\left(d_{0}\right) \\
\alpha \\
X_{\sigma}
\end{array}\right) .
\end{aligned}
$$

Equation (6) in matrix form can be written as

$$
S=A X,
$$

TABLE 1: Extracted parameters for the statistical path loss model [21].

\begin{tabular}{lccc}
\hline Implant to body surface & $\mathrm{PL}\left(d_{0}\right)$ & $\alpha$ & $\sigma$ \\
\hline Deep-tissue & 47.14 & 4.26 & 7.85 \\
Near surface & 49.81 & 4.22 & 6.81 \\
\hline
\end{tabular}

where

$$
\begin{aligned}
& S=\left(\begin{array}{c}
S(1, j) \\
S(2, j) \\
\vdots \\
S(M, j)
\end{array}\right), \\
& A=\left(\begin{array}{ccc}
1 & 10 \log _{10}\left(\frac{d(1, j)}{d_{0}}\right) & 1 \\
1 & 10 \log _{10}\left(\frac{d(2, j)}{d_{0}}\right) & 1 \\
\vdots & \vdots & \vdots \\
1 & 10 \log _{10}\left(\frac{d(M, j)}{d_{0}}\right) & 1
\end{array}\right),
\end{aligned}
$$$$
X=\left(\begin{array}{c}
\mathrm{PL}\left(d_{0}\right) \\
\alpha \\
X_{\sigma}
\end{array}\right) \text {. }
$$

The statistical path loss model for medical implant communication service (MICS) band has been developed by the National Institute of Science and Technology (NIST) [21]. The simulation system in [21] includes a three-dimensional human body model, propagation engine which is a threedimensional full-wave electromagnetic field simulator (i.e., HFSS 1), the 3D immersive and visualization platform, and finally an implantable (or body surface antenna). The 3D human body model includes frequency dependent dielectric properties of $300+$ parts in a male human body with a resolution of $2 \mathrm{~mm}$. The extracted path loss parameters reported in [21] are summarized in Table 1. Among the two scenarios, the deep-tissue implant scenario considers endoscopy capsule applications for upper stomach $(95 \mathrm{~mm}$ below body surface) and lower stomach (118 mm below body surface). The operating frequency in the study [21] has been chosen to be $403.5 \mathrm{MHz}$ which is the midpoint of the MICS frequency band.

We use the log-normal shadowing model as shown in (5) to model the path loss for deep-tissue implant scenario statistically using the extracted parameters as summarized in Table 1. Figure 2(a) shows the probability distribution function (PDF) of the random path loss deviation. Figure 2(b) shows the variation of scattered path loss as a function of distances $(50-800 \mathrm{~mm})$. As we can see from the figures the path loss is highly scattered around a mean due to the random deviation, $X_{\sigma}$. 


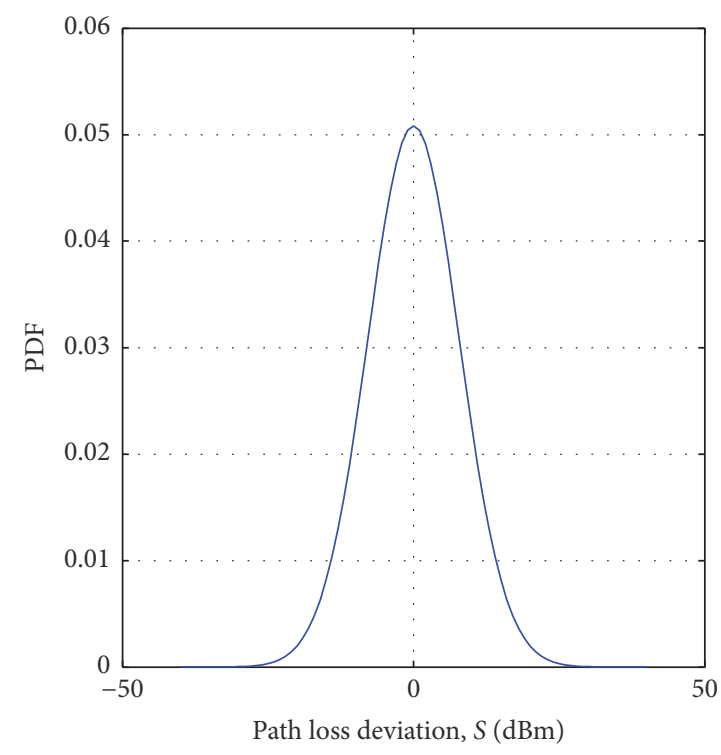

(a)

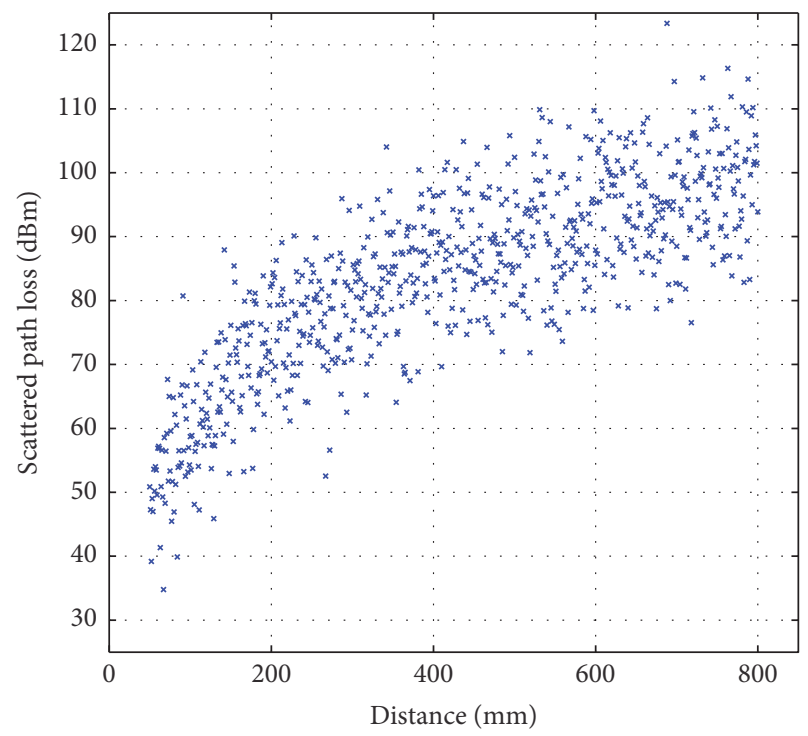

(b)

FIGURE 2: Statistical path loss model: (a) probability distribution function of path loss deviation; (b) path loss versus distance plot (deep-tissue implant scenario).

\section{Weighted Centroid Localization}

Centroid localization (CL) determines the centroid using $N$ position of the beacons within range as follows:

$$
E_{i}^{\prime}(x, y, z)=\frac{1}{N} \sum_{j=1}^{N} B_{j}(x, y, z) .
$$

Expressing $N$ as the sum of ones and multiplication of $B_{j}$ with ones, (9) is expanded to the weighted centroid localization (WCL) formula as follows:

$$
E_{i}^{\prime}(x, y, z)=\frac{1}{\sum_{j=1}^{N} 1} \sum_{j=1}^{N} 1 B_{j}(x, y, z) .
$$

Replacing ones by weight functions $W(i, j)$, the final equation of WCL is formed as

$$
E_{i}^{\prime \prime}(x, y, z)=\frac{1}{\sum_{j=1}^{N} W(i, j)} \sum_{j=1}^{N} W(i, j) B_{j}(x, y, z),
$$

where $W(i, j)$ is the weight of the $j$ th beacon at $i$ th position of the target.

\section{Proposed Algorithm}

In our proposed algorithm, the weight of the sensors position is inversely proportional to the $\mathrm{Tx}-\mathrm{Rx}$ separation distance $d(i, j)$. The distance $d(i, j)$ is calculated using the measured randomly scattered path loss. Then WCL is used to find the initial position. Finally, a calibration process is applied on the initial position to estimate the final position. We propose suboptimal method of coefficient calculation and also compute the optimal value of coefficient for comparison. The information flow of the proposed algorithm is shown in Figure 3. The pseudocode of scattered path loss based WCL for VCE localization is shown in Algorithm 1. The development steps of the proposed algorithm are discussed in detail in the following.

5.1. Extraction of Path Loss Parameters. The main challenge in calculating the distance using measured path loss is the log-normally distributed random deviation $X_{\sigma}\left(0, \sigma^{2}\right)$ which is due to the nonhomogeneous environment of radio propagation inside human body. To address the randomness issue, we propose a method of estimating the local path loss parameters $\left(m_{1}\right.$ and $\left.m_{2}\right)$ of the randomly scattered path loss using adaptive least square error (LSE) estimation. We divide the traveling location of the capsule into clusters of equal size. Each cluster consists of $k$ neighboring points of capsules location. We estimate the parameters for a cluster and gradually for all traveling points. Then we use these estimated parameters and the measured path loss in the test phase to find the distance to calculate the weight of sensors.

The proposed estimated path loss model is as follows:

$$
\operatorname{EPL}(i, j)=m_{1}(i, j)+10 m_{2}(i, j) \log _{10}\left(\frac{d(i, j)}{d_{0}}\right)
$$

where $m_{1}(i, j)$ and $m_{2}(i, j)$ are the local path loss parameters. For $M$ possible positions of the target, we can represent the estimated path loss as 


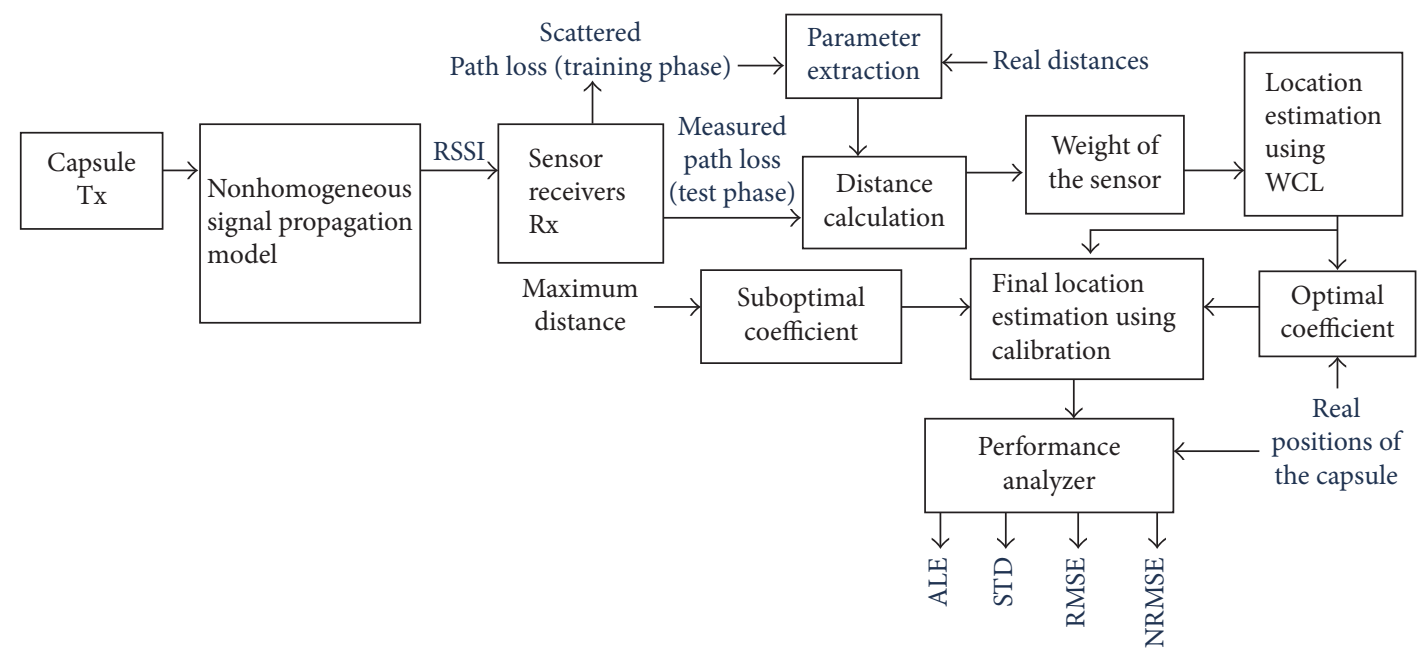

FIGURE 3: Information flow of optimal and proposed methods of scattered path loss based WCL.

$$
\begin{aligned}
& \left(\begin{array}{c}
\operatorname{EPL}(1, j) \\
\operatorname{EPL}(2, j) \\
\vdots \\
\operatorname{EPL}(M, j)
\end{array}\right) \\
& =\left(\begin{array}{cc}
1 & 10 \log _{10}\left(\frac{d(1, j)}{d_{0}}\right) \\
1 & 10 \log _{10}\left(\frac{d(2, j)}{d_{0}}\right) \\
\vdots & \vdots \\
1 & 10 \log _{10}\left(\frac{d(M, j)}{d_{0}}\right)
\end{array}\right)
\end{aligned}
$$

Equation (13) in matrix form can be written as

$$
\mathrm{EPL}=H \Theta,
$$

where

$$
\begin{aligned}
\operatorname{EPL} & =\left(\begin{array}{c}
\operatorname{EPL}(1, j) \\
\operatorname{EPL}(2, j) \\
\vdots \\
\operatorname{EPL}(M, j)
\end{array}\right), \\
H & =\left(\begin{array}{cc}
1 & 10 \log _{10}\left(\frac{d(1, j)}{d_{0}}\right) \\
1 & 10 \log _{10}\left(\frac{d(2, j)}{d_{0}}\right) \\
\vdots & \vdots \\
1 & 10 \log _{10}\left(\frac{d(M, j)}{d_{0}}\right)
\end{array}\right),
\end{aligned}
$$

$$
\Theta=\left(\begin{array}{l}
m_{1}(i, j) \\
m_{2}(i, j)
\end{array}\right) .
$$

The LSE is found [33] by minimizing the square error between the scattered path loss and the estimated path loss as

$$
\begin{aligned}
J(\Theta) & =\sum_{i=1}^{M}(S(i, j)-\operatorname{EPL}(i, j))^{2} \\
& =(S-H \Theta)^{T}(S-H \Theta) .
\end{aligned}
$$

In this paper, we propose a method of finding local path loss parameters of a cluster of $k$ neighboring locations in the training phase using adaptive LSE as follows:

Determine: $\theta$

To Minimize: $J(\theta)$

$$
\begin{aligned}
& =\sum_{m}^{m+k-1}(S(m, j)-\operatorname{EPL}(m, j))^{2} \\
& =(\Lambda-\hbar \theta)^{T}(\Lambda-\hbar \theta),
\end{aligned}
$$

where $m$ represents the capsule's position, $k$ is the number of neighboring target positions in a cluster, $\Lambda$ is a $k \times 1$ matrix containing the scattered path loss from $k$ neighboring positions of the capsule to sensor $j, \hbar$ is the distance matrix of $k \times 2$ target positions from sensor $j$, and $\theta$ is a matrix containing local path loss parameters $m_{1}(i, j)$ and $m_{2}(i, j)$ for any specific cluster. Equation (17) can be rearranged as

$$
\begin{aligned}
J(\theta) & =\left(\Lambda^{T} \Lambda-\Lambda^{T} \hbar \theta-\hbar^{T} \theta^{T} \Lambda+\hbar^{T} \theta^{T} \hbar \theta\right) \\
& =\left(\Lambda^{T} \Lambda-2 \Lambda^{T} \hbar \theta+\hbar^{T} \theta^{T} \hbar \theta\right) .
\end{aligned}
$$

The gradient is

$$
\frac{\partial J(\theta)}{\partial \theta}=-2 \hbar^{T} \Lambda+2 \hbar^{T} \hbar \theta
$$




\section{Training Phase:}

\{\% Local path loss parameter $m_{1}$ and $m_{2}$ estimation\} Initialization: $k=3, i=0, \Lambda=0, \hbar=0$ Input: $\operatorname{PL}\left(d_{0}\right), \alpha, X_{\sigma}, d_{0}, R_{i}, B_{j}[\forall i, \forall j]$

Output: $m_{1}, m_{2}$

while $i<M$ do

$m=i+1$

for $j=1,2, \ldots, N$ do

for $i=m, \ldots, m+k-1$ do

$d(i, j)=\sqrt{\left(R_{i}-B_{j}\right)^{2}}$

$\{\%$ Scattered Path loss for a cluster $\}$

$\Lambda(i, 1)=\operatorname{PL}\left(d_{0}\right)+10 \alpha \log _{10}\left(\frac{d(i, j)}{d_{0}}\right)+X_{\sigma}$

$\hbar(i,:)=\left[\begin{array}{ll}1 & \left.10 \log _{10}\left(\frac{d(i, j)}{d_{0}}\right)\right]\end{array}\right.$

\section{end for}

$\theta=\left(\hbar^{T} \hbar\right)^{-1} \hbar^{T} \Lambda$

for $i=m, \ldots, m+k-1$ do

$m_{1}(i, j)=\theta(1)$

$m_{2}(i, j)=\theta(2)$

end for

end for

end while

Test Phase:

Initialization: $k=3, i=0$

Input: $\operatorname{PL}\left(d_{0}\right), \alpha, X_{\sigma}, R, d_{0}, d_{\max }, B_{j}[\forall j]$

Output: $E, P$

\{\% Initial position $E$ estimation $\}$

while $i<M$ do

$m=i+1$

for $i=m, \ldots, m+k-1$ do

for $j=1,2, \ldots, N$ do

$\{\%$ Scattered Measured Path loss modeling\}

$\mathrm{PL}(i, j)=\mathrm{PL}\left(d_{0}\right)+10 \alpha \log _{10}\left(\frac{d(i, j)}{d_{0}}\right)+X_{\sigma}$

\{\% Distance estimation using $m_{1}$ and $m_{2}$ \}

$d(i, j)=d_{0} 10^{\left(\mathrm{PL}(i, j)-m_{1}(i, j)\right) / 10 m_{2}(i, j)}$

\{\% Weight calculation $\}$

$W(i, j)=\frac{1}{d(i, j)}$

end for

$\{\%$ Position estimation using WCL $\}$

$E_{i}\left(x_{e}, y_{e}, z_{e}\right)=\frac{\sum_{j=1}^{N}\left(W(i, j) B_{j}\left(x_{j}, y_{j}, z_{j}\right)\right)}{\sum_{j=1}^{N} W(i, j)}$

end for

end while

\{\% Optimal and sub-optimal coefficient

$c_{\mathrm{opt}}=\left(E E^{T}\right)^{-1} R E^{T}$

$c_{\text {sopt }}=\log _{10}\left(d_{\text {max }}\right)$

\{\% Final position $P$ estimation $\}$

for $i=1,2, \ldots, M$ do

$\{\%$ Optimal method of calibration $\}$

$P_{i}(x, y, z)=c_{\text {opt }} E_{i}$.

$\{\%$ Proposed method of calibration $\}$

$P_{i}(x, y, z)=c_{\text {sopt }} E_{i}$.

end for

Algorithm 1: Optimal and proposed methods of scattered path loss based WCL. 
Setting the gradient equal to zero yields the adaptive LSE estimate of local path loss parameters:

$$
\theta=\left(\hbar^{T} \hbar\right)^{-1} \hbar^{T} \Lambda
$$

5.2. Distance Estimation. The extracted parameters in the training phase as shown in (20) are used to estimate the distance of the target from the sensors in the test phase. In the test phase, we obtain the randomly scattered path loss $\operatorname{PL}(i, j)$ using the measured RSSI and the transmitted power as shown in (2) which can be expressed as

$$
\mathrm{PL}(i, j)=\operatorname{PL}\left(d_{0}\right)+10 \alpha \log _{10}\left(\frac{d(i, j)}{d_{0}}\right)+X_{\sigma} .
$$

Then, we calculate the distance $d(i, j)$ of the target from the sensors using the randomly scattered measured path loss $\operatorname{PL}(i, j)$ and the extracted local parameters $\left(m_{1}(i, j)\right.$ and $\left.m_{2}(i, j)\right)$ as follows:

$$
d(i, j)=d_{0} 10^{\left(\mathrm{PL}(i, j)-m_{1}(i, j)\right) / 10 m_{2}(i, j)} .
$$

It is to noted that $\operatorname{PL}(i, j)$ is absolutely different than the scattered path loss $S(i, j)$ used in the training phase.

5.3. Location Estimation. The distance estimated using (22) is used to calculate the weight of the sensors as follows:

$$
W(i, j)=\frac{1}{d(i, j)}
$$

The position of the capsule is initially estimated using traditional WCL as follows:

$$
\begin{aligned}
E_{i}\left(x_{e}, y_{e}, z_{e}\right) & =\frac{\sum_{j=1}^{N}\left(W(i, j) B_{j}\left(x_{j}, y_{j}, z_{j}\right)\right)}{\sum_{j=1}^{N} W(i, j)} \\
& =\frac{\sum_{j=1}^{N}\left((1 / d(i, j)) B_{j}\left(x_{j}, y_{j}, z_{j}\right)\right)}{\sum_{j=1}^{N}(1 / d(i, j))},
\end{aligned}
$$

where $B_{j}$ is the fixed known position of the sensor nodes. Then, we can apply a calibration process as in [18] to estimate the final position $P_{i}(x, y, z)$ more accurately as follows:

$$
P_{i}(x, y, z)=c E_{i}\left(x_{e}, y_{e}, z_{e}\right)
$$

where $c$ is the calibration coefficient. We propose a suboptimal method of estimation of the calibration coefficient and also compute the optimal value of coefficient analytically to compare the performance of the proposed suboptimal method.

\subsubsection{Analytical Method of Optimal Coefficient Computa-} tion. We can compute the optimal value of the calibration coefficient by minimizing the mean square error (MSE) of the final estimated positions compared to the real positions
$\left(R_{i}\right)$. The problem of determining the optimal coefficient $c_{\mathrm{opt}}$ minimizing MSE is formulated as follows:

Determine: $c_{\mathrm{opt}}$

$$
\text { To Minimize: } \begin{aligned}
\text { MSE } & =\frac{\sum_{i=1}^{M}\left(R_{i}-P_{i}\right)^{2}}{M} \\
& =\frac{(R-c E)^{T}(R-c E)}{M} \\
& =\frac{1}{M}\left(R^{T} R-R^{T} E c-E^{T} c^{T} R+E^{T} c^{T} E c\right) \\
& =\frac{1}{M}\left(R^{T} R-2 R^{T} E c+H^{T} c^{T} H c\right),
\end{aligned}
$$

where $M$ is the number of locations, $P_{i}$ is the final estimated position, $E$ is the initial estimated position in matrix form, and $R$ is the real position of the capsule in matrix form. Differentiating MSE with respect to $c$, we find

$$
\frac{d(\mathrm{MSE})}{d c}=-2 E^{T} R+2 E^{T} E c
$$

Equating first derivative to zero yields

$$
c_{\mathrm{opt}}=\left(E E^{T}\right)^{-1} R E^{T} .
$$

Replacing $c_{\text {opt }}$ in (25), the final estimated position is found as

$$
P_{i}(x, y, z)=c_{\mathrm{opt}} E_{i}
$$

5.3.2. Suboptimal Method. We propose a suboptimal method of finding calibration coefficient using the logarithm of the maximum distance $d_{\max }$ as

$$
c_{\text {sopt }}=\log _{10}\left(d_{\text {max }}\right) \text {. }
$$

Using (25), the final estimated position is found as

$$
P_{i}(x, y, z)=c_{\text {sopt }} E_{i} .
$$

The optimal value of coefficient is dependent on the real positions of capsule, whereas the suboptimal method does not require any prior knowledge of real positions. Since the dimension of the sensor network and the dimension of the region of interest (ROI) are fixed, the maximum covered range $d_{\max }$ is a known value. Thus, it is realistic to calculate the coefficient using suboptimal method.

In next section, we will see the performances of the proposed algorithm.

\section{Performance Indices}

The localization accuracy is analyzed using the performance indices such as localization error (LE), average localization error (ALE), root mean square error (RMSE), and the standard deviation of error (STD). The RMSE is normalized to find NRMSE to compare the results for different scales. 


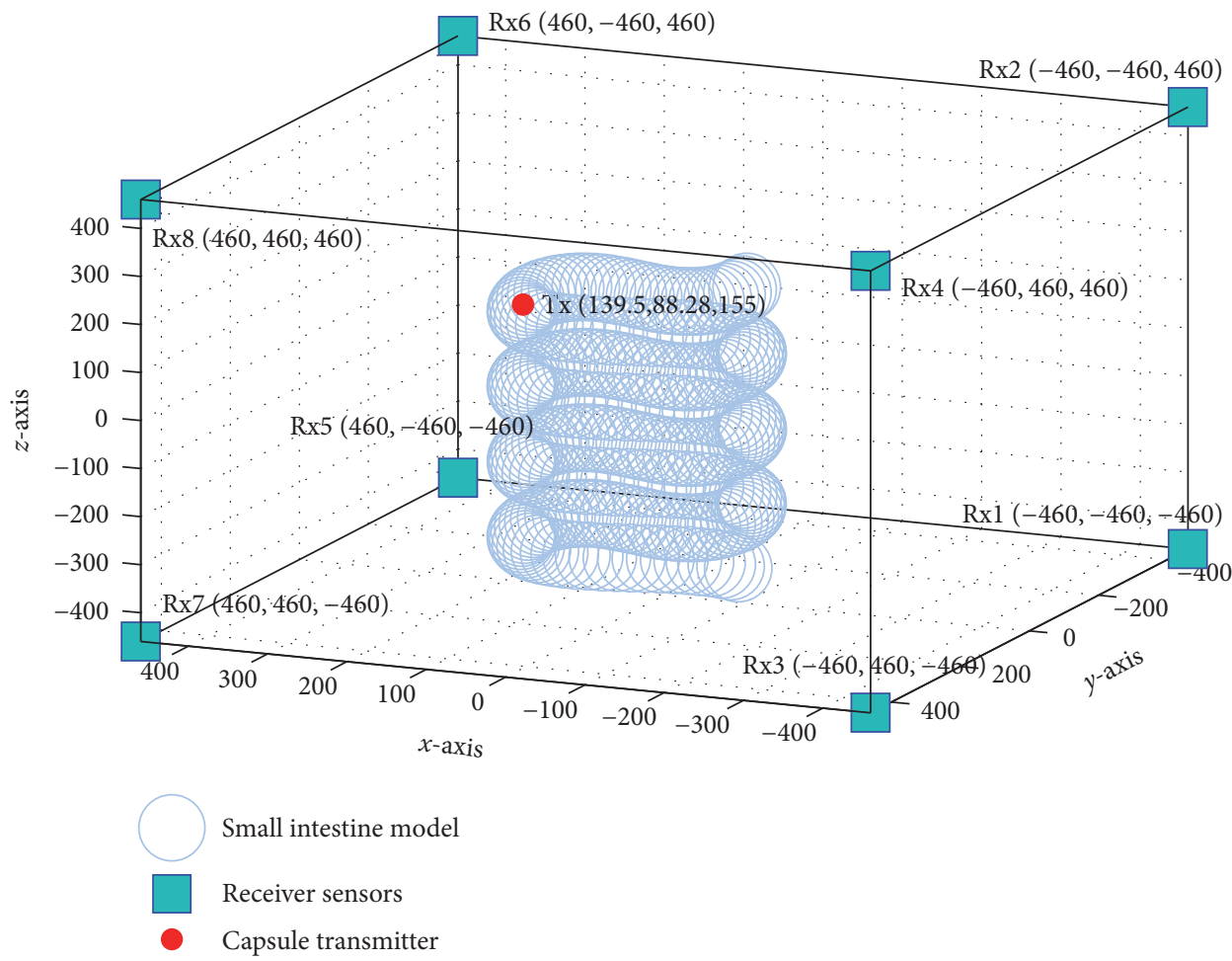

FIGURE 4: Simulation system.

Localization error (LE) is expressed as the difference between estimated and real positions as

$$
\begin{aligned}
\mathrm{LE}_{i} & =\sqrt{\left(R_{i}-P_{i}\right)^{2}} \\
& =\sqrt{\left(x_{r}-x_{e}\right)^{2}+\left(y_{r}-y_{e}\right)^{2}+\left(z_{r}-z_{e}\right)^{2}},
\end{aligned}
$$

where $P_{i}$ is the estimated position using proposed algorithm and $R_{i}$ is the real position of the capsule. $\left(x_{e}, y_{e}, z_{e}\right)$ is the coordinate of the estimated position and $\left(x_{r}, y_{r}, z_{r}\right)$ is the coordinate of the real position. For $M$ simulation results of $M$ sample positions, the average localization error (ALE) is calculated as follows:

$$
\mathrm{ALE}=\frac{\sum_{i=1}^{M} \mathrm{LE}_{i}}{M} .
$$

For $M$ sample positions, root mean square error (RMSE) is calculated as follows:

$$
\mathrm{RMSE}=\sqrt{\frac{\sum_{i=1}^{M}\left(R_{i}-P_{i}\right)^{2}}{M}}=\sqrt{\frac{\sum_{i=1}^{M} \mathrm{LE}_{i}^{2}}{M}} .
$$

The standard deviation of error is expressed as

$$
\mathrm{STD}=\sqrt{\frac{\sum_{i=1}^{M}\left(\mathrm{LE}_{i}-\mathrm{ALE}\right)^{2}}{M}} .
$$

The normalized error (in \%) is found as follows:

$$
\text { Normalized error }(\%), \text { NRMSE }=\frac{\text { RMSE }}{\sqrt{\left(R_{\max }-R_{\min }\right)^{2}}},
$$

where $R_{\max }$ is the maximum value of coordinates in $x-y-z$ direction and $R_{\min }$ is the minimum value of coordinates in $x-y-z$ direction.

\section{Simulation and Results}

As experimentation using real human body is not possible due to practical limitation, we develop a $3 \mathrm{D}$ simulation platform of VCE localization using MATLAB to verify the accuracy of our proposed algorithm considering the real radio propagation characteristics of human body channel. The simulation system as shown in Figure 4 includes the randomly scattered statistical path loss model of human body channel, a sensor network of $920 \mathrm{~mm} \times 920 \mathrm{~mm} \times 920 \mathrm{~mm}$ dimension, and a small intestine model of $280 \mathrm{~mm} \times 120 \mathrm{~mm} \times$ $320 \mathrm{~mm}$ dimension. The path loss of the communication channel between the capsule and the sensors is statistically modeled using the path loss parameters of deep-tissue implant scenario [21] as summarized in Table 1 as shown in (6). The path loss model is scattered as shown in Figure 2(b) due to the zero-mean Gaussian random variable $X_{\sigma}$ with variance $\sigma^{2}$. The position of the capsule is indicated using red marker in Figure 3. The real position of the capsule is mapped using MATLAB. It can simulate the proposed algorithm and shows the estimated as well as real positions in the same $3 \mathrm{D}$ visualization platform.

The simulation is carried out in the training phase and test phase which are explained in the following.

7.1. Training Phase. In the training phase, we model the path loss for 2529 target positions of the capsule in the training 


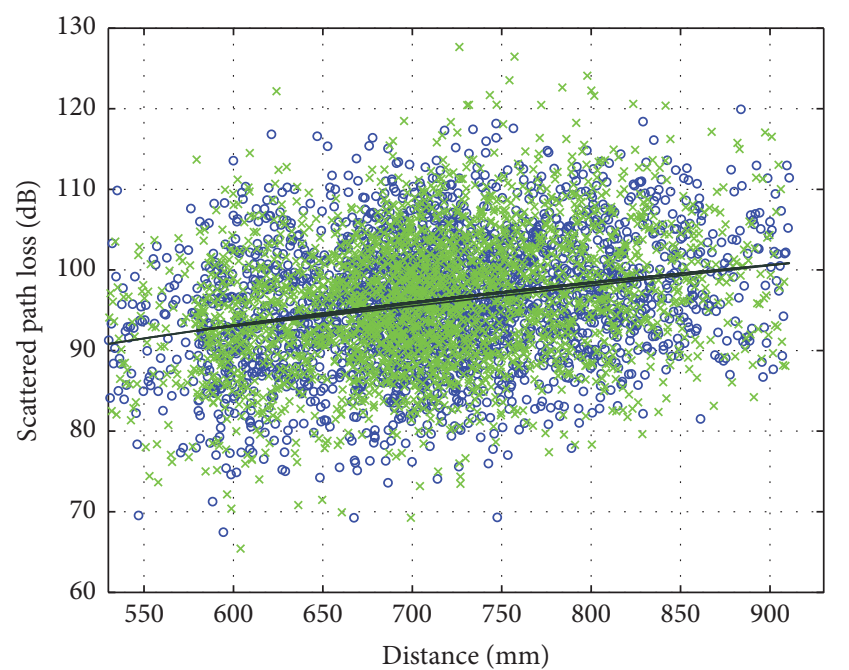

- Measured path loss (test phase)

$\times \quad$ Scattered path loss (training phase)

_ Mean path loss

FigURE 5: Scattered path loss as a function of distance.

phase using $\alpha$ equal to $4.16, \operatorname{PL}\left(d_{0}\right)$ equal to 47.14 , and $\sigma$ equal to 7.85 as shown in Table 1 for deep-tissue scenario which has been plotted as a function of distance in Figure 5. Then we use the scattered path loss of $k$ neighboring target positions and the real distances to extract local path loss parameters $\left(m_{1}(i, j)\right.$ and $\left.m_{2}(i, j)\right)$ using adaptive LSE method. For our system, we set the value of $k$ as 3 . We model the estimated path loss using the extracted parameters. The extracted parameters in the training phase are used in the test phase to calculate the distance using the measured path loss.

7.2. Test Phase. We generate path loss data using Table 1 deeptissue scenario statistics again. However, now we keep the distances of capsule from sensor nodes same as it is in training phase to show the best possible performance of our proposed algorithm. It is noted that at the end of this section we also evaluate performance of our proposed algorithm for the other cases when path loss is generated using randomly deviated distances of capsule from sensor nodes compared to training phase.

Figure 5 compares the measured path loss in the test phase (for 2529 possible target positions) to the scattered path loss of the training phase. As we can see in Figure 5 the randomness of the measured path loss in the test phase is not similar to that in the training phase. Figure 6 shows the comparison of estimated path loss and the measured path loss for 30 sample target locations. Figure 7 compares the real and estimated distances of 30 target positions. As we can see the estimated distances are in good agreement with the real distances. Finally, we estimate the position of the target using WCL considering the corresponding estimated distances as the weight of the sensors positions. We use different performance indices as ALE, RMSE, STD, and NRMSE to verify the accuracy of our proposed algorithms. To analyze the impact of the number of sensors and the deployment topology on

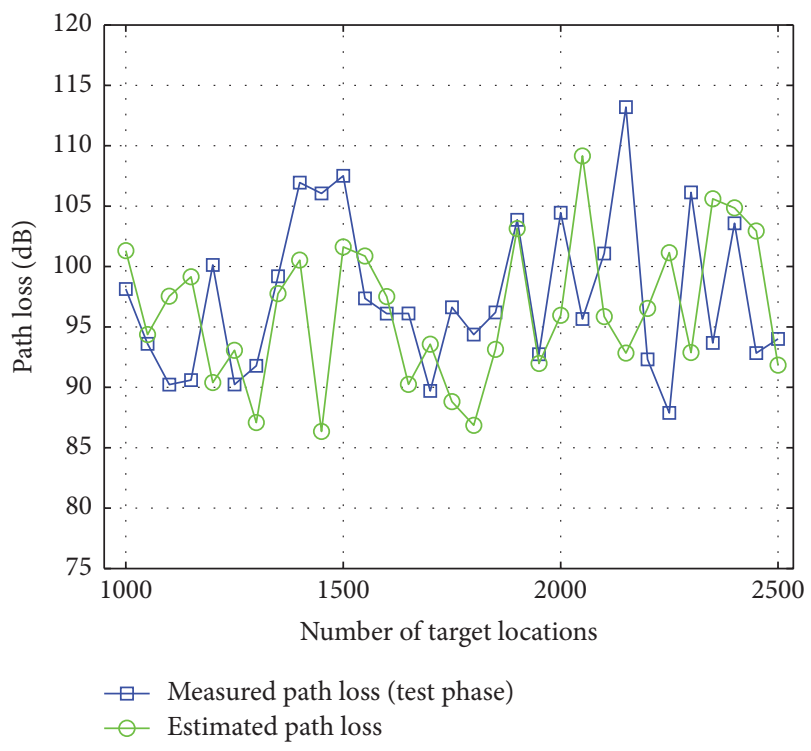

Figure 6: Measured and estimated path loss for 30 sample target positions.

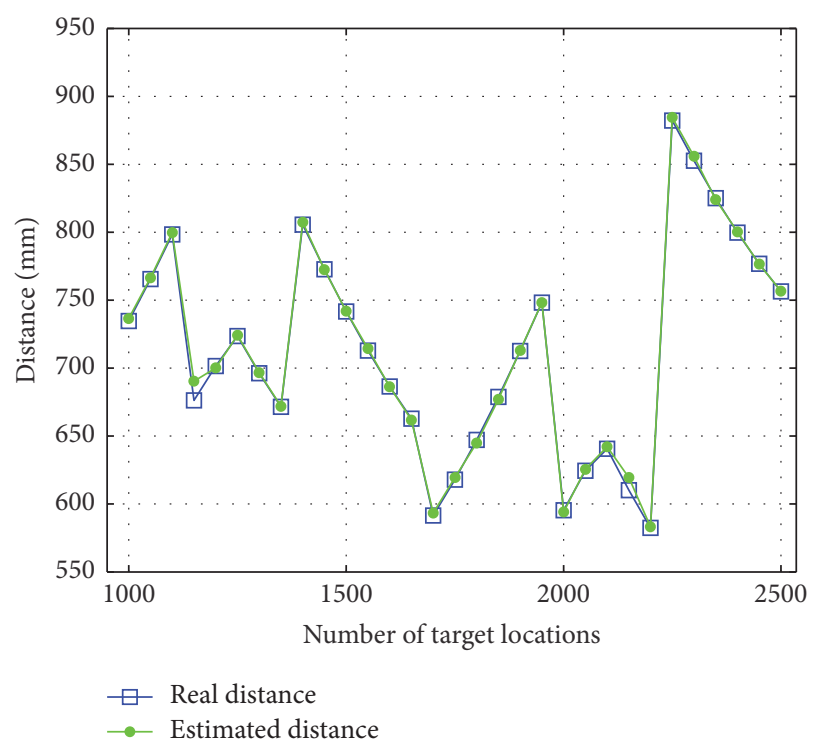

FIGURE 7: Real and estimated distance for 30 sample target positions.

the localization accuracy, we gradually increase the number of sensors as 16, 24, 32, 40, 48, 56, and 64 and follow three different topologies of sensor deployment. Figure 8 shows the three different topologies of sensor deployment using 64 sensors. Figures 9(a) and 9(b) show the impact of sensor topology on localization accuracy. As we can see the best accuracy is obtained using topology 1 . Using topology 2 , the accuracy is moderate and using topology 3 the ALE as well as the STD is very high. Figures 10 (a) and 10(b) show the impact of the number of sensors on localization accuracy. As we can see the accuracy improves about $2 \mathrm{~mm}$ for each 16 additional sensors. Figures 9 and 10 show that the best localization accuracy is obtained using topology 1 and 64 sensors. 


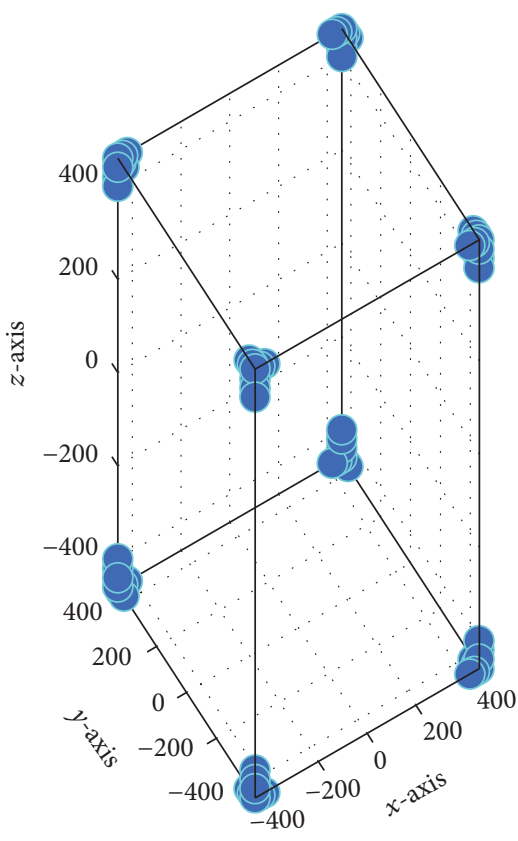

Topology 1

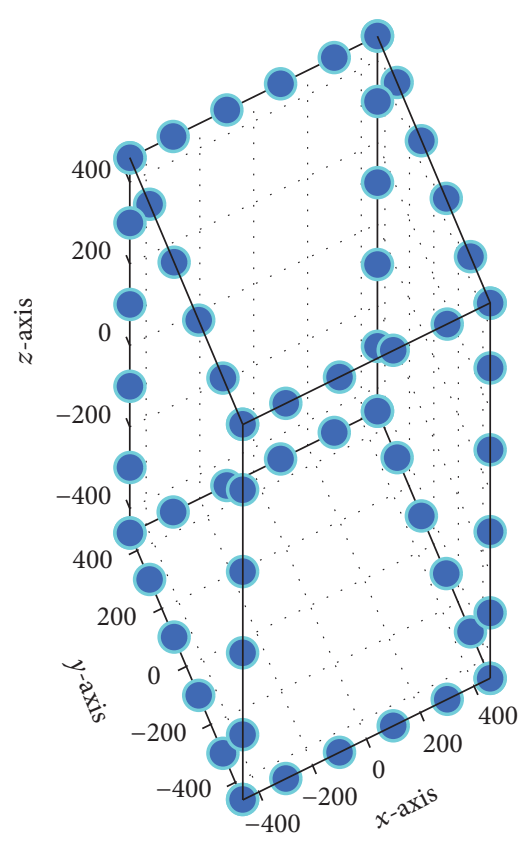

Topology 2

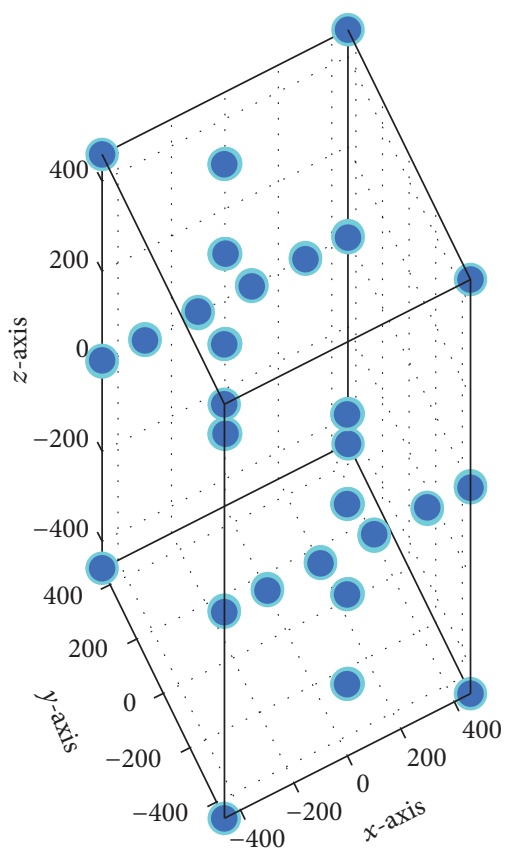

Topology 3

FIGURE 8: Sensors topology.

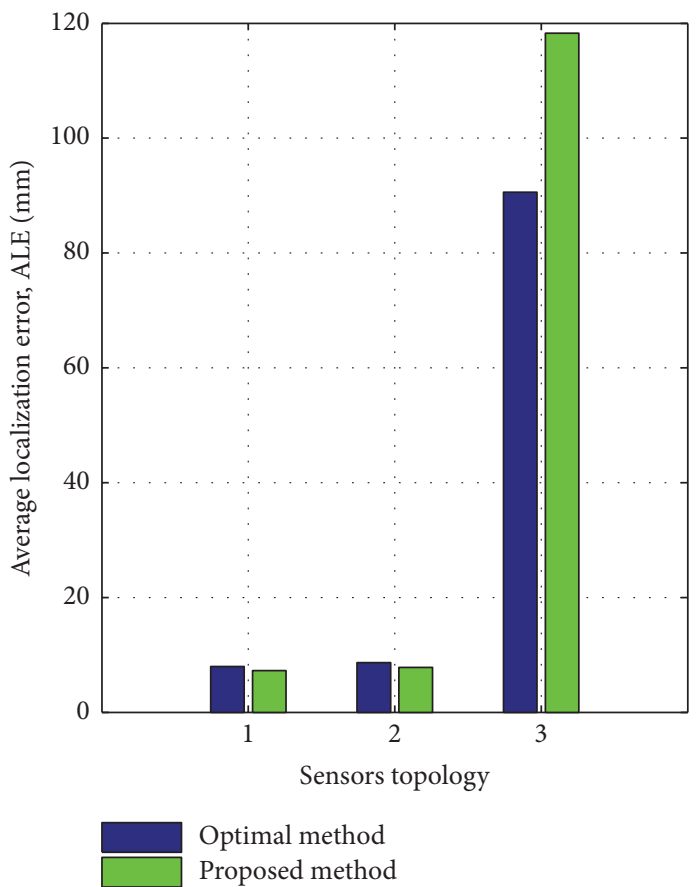

(a)

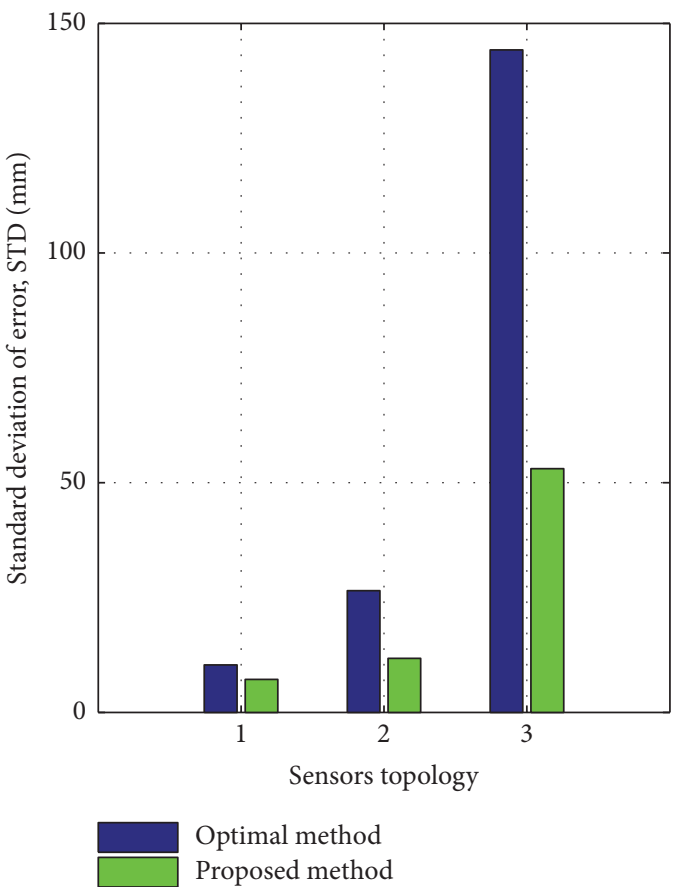

(b)

FIGURE 9: Impact of sensors topology on localization accuracy: (a) average localization error, ALE; (b) standard deviation of error, STD.

Keeping sensor topology 1 and number of sensors as 64, next we simulate both proposed and optimal methods of scattered path loss based WCL for eight different dimensions of the sensor network to compare their accuracy. Table 2 presents the performance of both of the methods as well as that of traditional WCL for different network dimension. As we can see from Table 2, the average localization error of the traditional WCL is very high and the calibration process in our proposed algorithm improves the accuracy of localization significantly. The results also show that the 


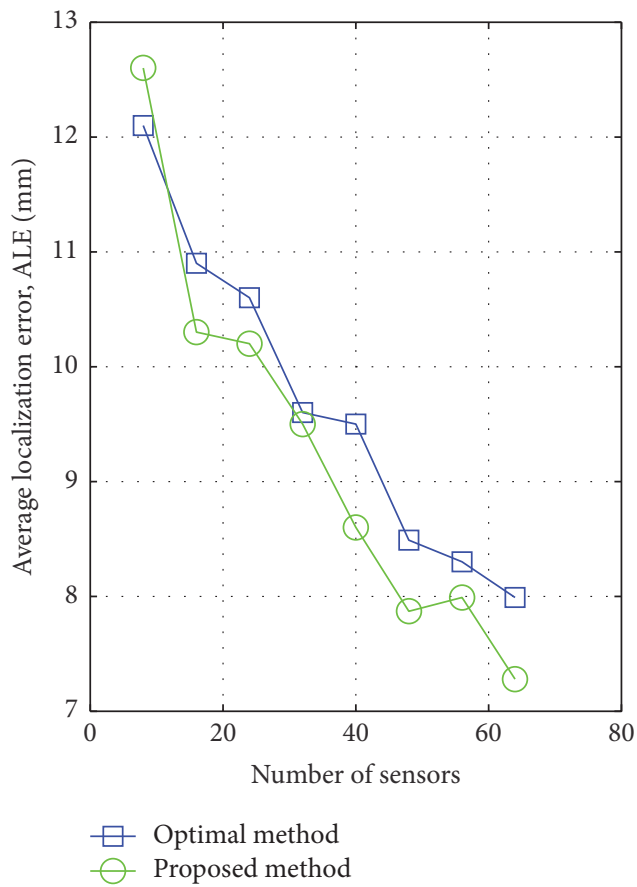

(a)

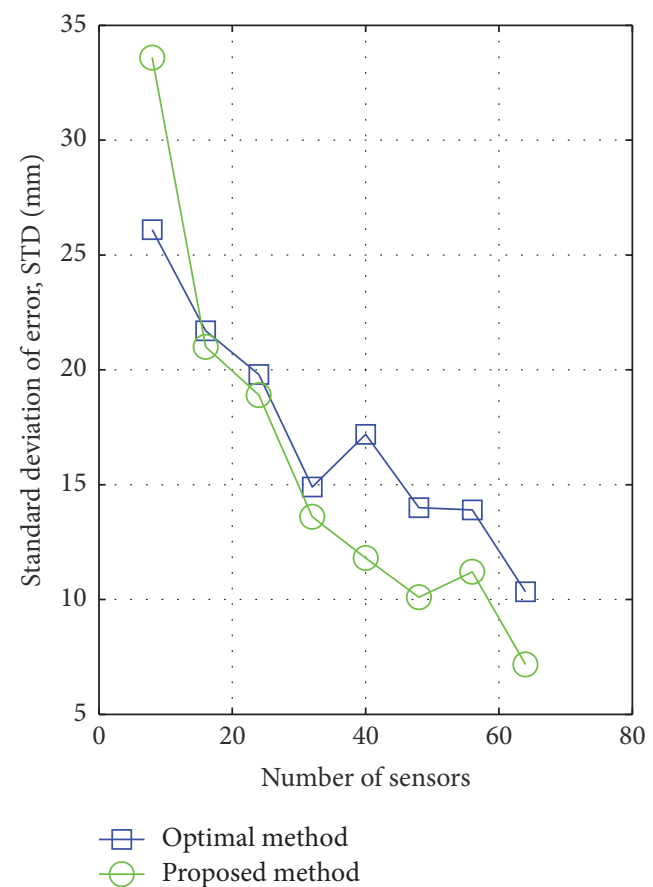

(b)

FIGURE 10: Impact of the number of sensors on localization accuracy: (a) average localization error, ALE; (b) standard deviation of error, STD.

TABLE 2: Accuracy of different optimization methods for different dimension of the network.

\begin{tabular}{|c|c|c|c|c|}
\hline \multirow[b]{2}{*}{$\begin{array}{l}\text { Sensor network } \\
\text { dimension (in } \mathrm{mm} \text { ) }\end{array}$} & \multicolumn{3}{|c|}{ Average localization error (ALE, in $\mathrm{mm}$ ) using traditional and proposed WCL } & \multirow[b]{2}{*}{$\begin{array}{c}\text { Scattered path loss } \\
\text { based proposed WCL, } \\
\qquad W=\frac{1}{d(i, j)}\end{array}$} \\
\hline & $\begin{array}{l}\text { Scattered path loss based } \\
\text { traditional WCL, } \\
\qquad W=\frac{1}{\mathrm{PL}(i, j)}\end{array}$ & $\begin{array}{l}\text { Scattered path loss based } \\
\text { traditional WCL, } \\
\qquad W=\frac{1}{d(i, j)}\end{array}$ & $\begin{array}{l}\text { Scattered path loss based } \\
\text { optimal WCL } \\
\text { (benchmark), } \\
W=\frac{1}{d(i, j)}\end{array}$ & \\
\hline $600 \times 600 \times 600$ & 139.30 & 98.20 & 12.00 & 10.00 \\
\hline $680 \times 680 \times 680$ & 140.26 & 98.43 & 10.00 & 9.00 \\
\hline $760 \times 760 \times 760$ & 140.93 & 98.59 & 9.00 & 8.00 \\
\hline $840 \times 840 \times 840$ & 141.40 & 98.70 & 9.00 & 7.80 \\
\hline $920 \times 920 \times 920$ & 141.09 & 98.78 & 7.99 & 7.28 \\
\hline $1000 \times 1000 \times 1000$ & 141.33 & 98.84 & 8.10 & 8.39 \\
\hline $1040 \times 1040 \times 1040$ & 142.25 & 98.87 & 8.00 & 8.25 \\
\hline $1080 \times 1080 \times 1080$ & 142.29 & 98.89 & 8.40 & 8.70 \\
\hline
\end{tabular}

optimal benchmark accuracy is possible to be achieved using our proposed suboptimal method and for each dimension of the network the proposed method performs equally well. As we can see, using both optimal and proposed methods, best localization accuracy is found for $920 \mathrm{~mm} \times 920 \mathrm{~mm} \times$ $920 \mathrm{~mm}$ dimension of the network. Thus, we simulate the proposed algorithms using 64 sensors to localize the capsule at 2529 target positions in the small intestine by using sensor topology 1 and $920 \mathrm{~mm} \times 920 \mathrm{~mm} \times 920 \mathrm{~mm}$ dimension of the network.

Figure 11 shows the simulation results of position estimation using optimal coefficient based scattered path loss WCL. The estimated and real positions of 12 sample target are shown in Figure 11 and are summarized in Table 3. The simulation results using suboptimal coefficient based proposed algorithm are shown in Figure 12 and are summarized in Table 3. Figures 11 and 12 and Table 3 show that the estimated positions are in good agreement with the real positions.

Table 4 compares the proposed algorithm to other works in the literature $[10-15,17-19]$. It is to be noted that the works in $[18,19]$ are based on some assumptions on channel condition considering minimum path loss deviations which is not realistic for practical scenario of human body. Practically, the path loss is highly scattered due to the random path loss deviations as shown in (5) which is caused by the shadowing or multipath propagation effects of nonhomogeneous 


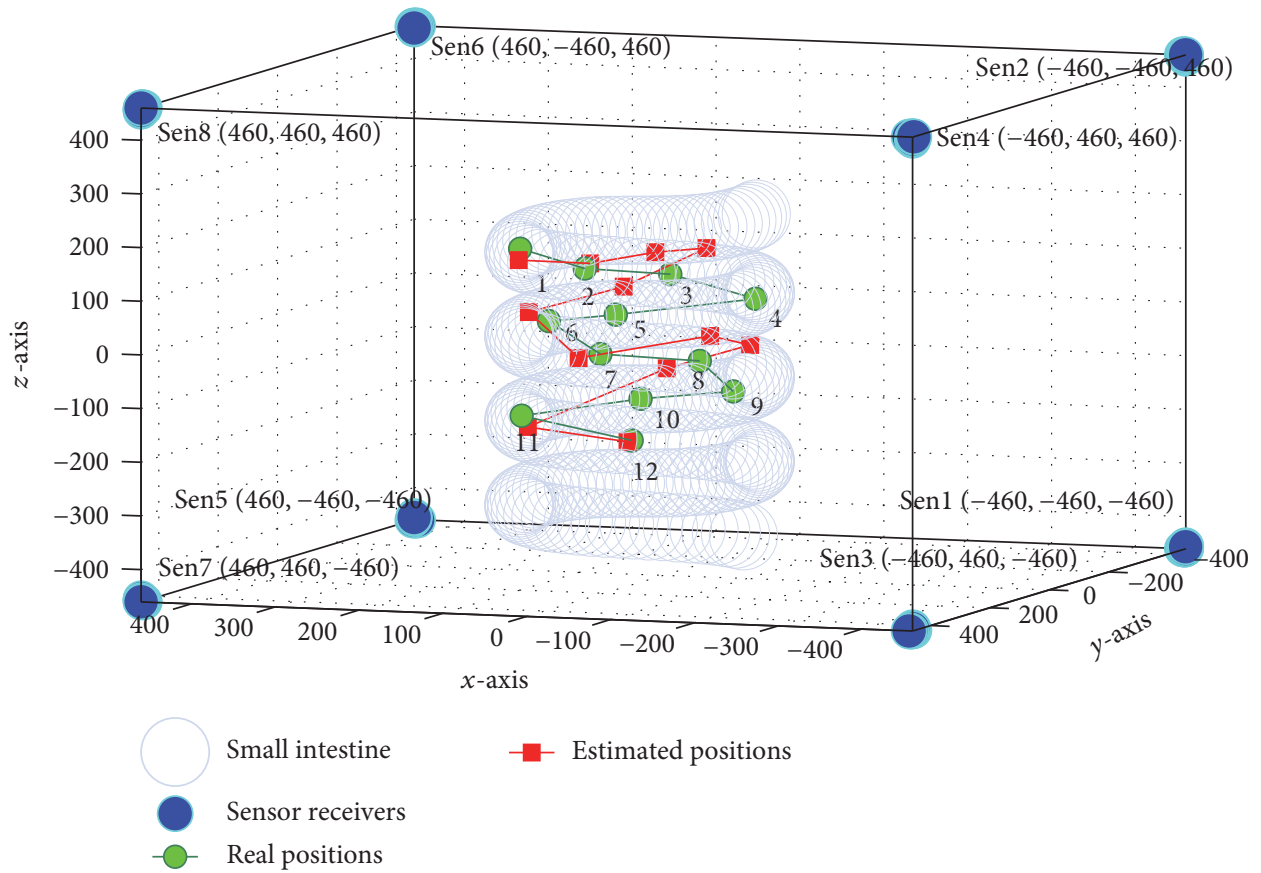

FIGURE 11: Simulation results using optimal method of scattered path loss based WCL.

TABLE 3: Real and estimated positions using optimal and proposed method of scattered path loss based WCL.

\begin{tabular}{lcc}
\hline Real positions, $R_{i}\left(x_{r}, y_{r}, z_{r}\right)$ & Estimated position using optimal method & Estimated position using proposed method \\
$P_{i}\left(x_{e}, y_{e}, z_{e}\right)$ & $\left.y_{e}, z_{e}\right)$ \\
\hline$(139.50,88.20,155.00)$ & $(142.60,84.10,132.90)$ & $(124.10,96.80,181.90)$ \\
$(52.90,115.00,127.50)$ & $(52.40,98.00,134.30)$ & $(25.60,93.20,166.00)$ \\
$(-29.51,59.39,112.50)$ & $(-0.12,27.28,146.60)$ & $(-4.19,150.40,178.30)$ \\
$(-139.90,84.10,77.50)$ & $(-84.14,92.54,170.00)$ & $(-124.20,115.80,197.10)$ \\
$(37.40,54.20,32.50)$ & $(44.00,9.00,76.80)$ & $(167.10,5.80,-23.51 .70)$ \\
$(122.90,37.90,12.50)$ & $(154.20,17.40,24.20)$ & $(53.90,82.60,-60)-23.70)$ \\
$(37.40,105.80,-32.50)$ & $(91.00,28.00,-55.70)$ & $(-82.40,71.70,-65.00)$ \\
$(-58.30,42.20,-50)$ & $(-70.40,41.46,-2.99)$ & $(-119.90,144.20,-19.20)$ \\
$(-125.50,119.70,-90.00)$ & $(-146.70,122.10,-1.80)$ & $(48.20,96.10,-124.40)$ \\
$(-1.70,81.20,-117.50)$ & $(-1.14,-7.88,-75.60)$ & $(119.50,104.70,-130.20)$ \\
$(140.00,81.60,-157.50)$ & $(113.20,136.50,-167.40)$ & $(-17.20,138.40,-207.40)$ \\
$(7.50,85.40,-195.00)$ & $(11.40,88.40,-197.20)$ & \\
\hline
\end{tabular}

medium of human body. Thus, the presented accuracy in [18, 19] considering ideal channel conditions is the best possible accuracy or the benchmark of the algorithms. However, to consider realistic channel conditions, we simulate the works in $[18,19]$ considering randomly scattered measured path loss to observe the effects of randomness on the accuracy and include the achievable results in Table 4 . We can see that due to randomness the localization errors of $[18,19]$ are extremely high in terms of RMSE and other performance indices. Table 4 also shows that using randomly scattered path loss the optimal coefficient based proposed benchmark of the algorithm can achieve RMSE of $13.17 \mathrm{~mm}$ while our proposed suboptimal coefficient based method can achieve RMSE $10.43 \mathrm{~mm}$ which is very close to the optimal benchmark accuracy. We may conclude from the comparison and results in Table 4 that our proposed algorithm can localize the capsule with significantly improved accuracy using randomly scattered path loss and outperforms the existing literature.

Considering the fact that the parameter estimation and path loss model may not be sufficiently accurate due to complex environment of experimentation and different human body structure, we generate path loss for both phases (training phase and test phase) using different random deviations of path loss and obtain RMSE of $10.43 \mathrm{~mm}$. Again, considering the fact that the target positions of capsule in the test phase may not be exactly same as those in the training phase, we generate path loss data considering random deviations in target movement positions as well (Gaussian random 


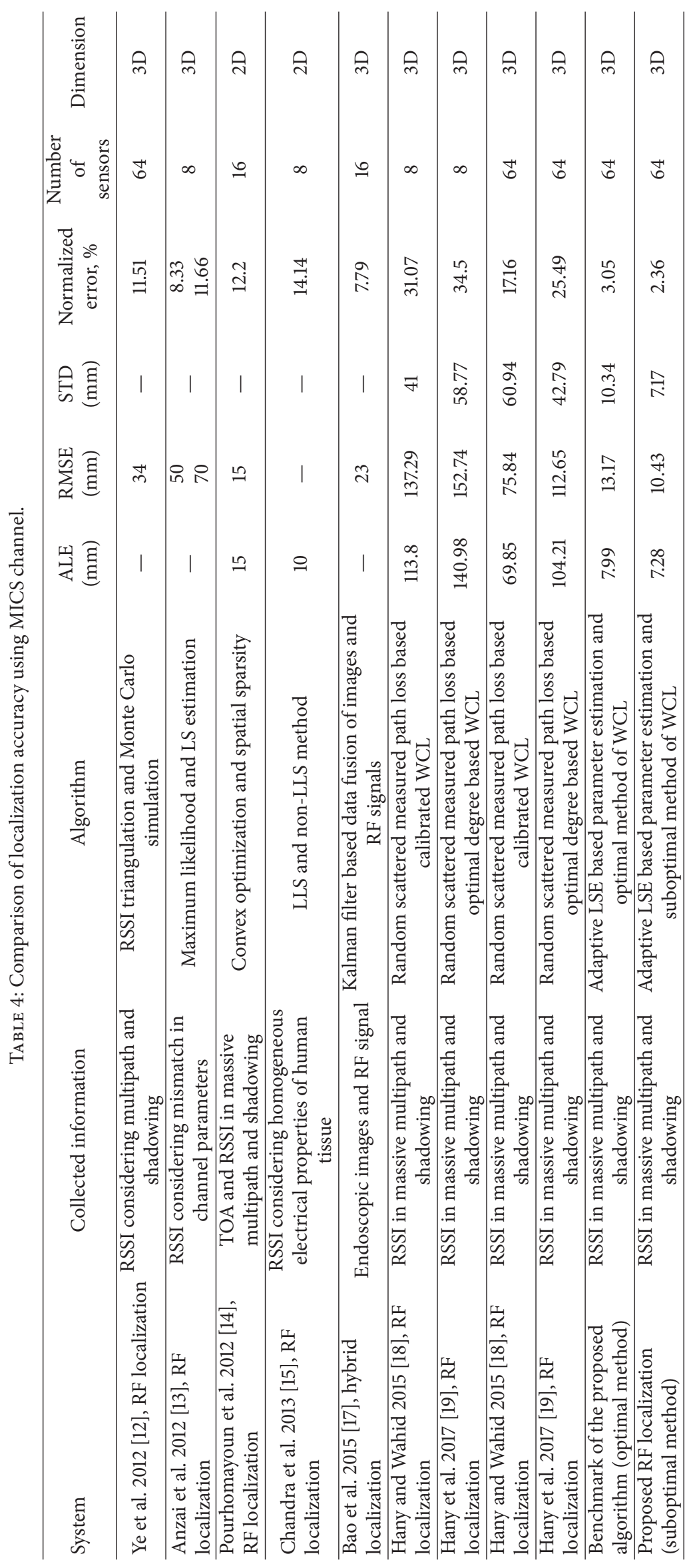




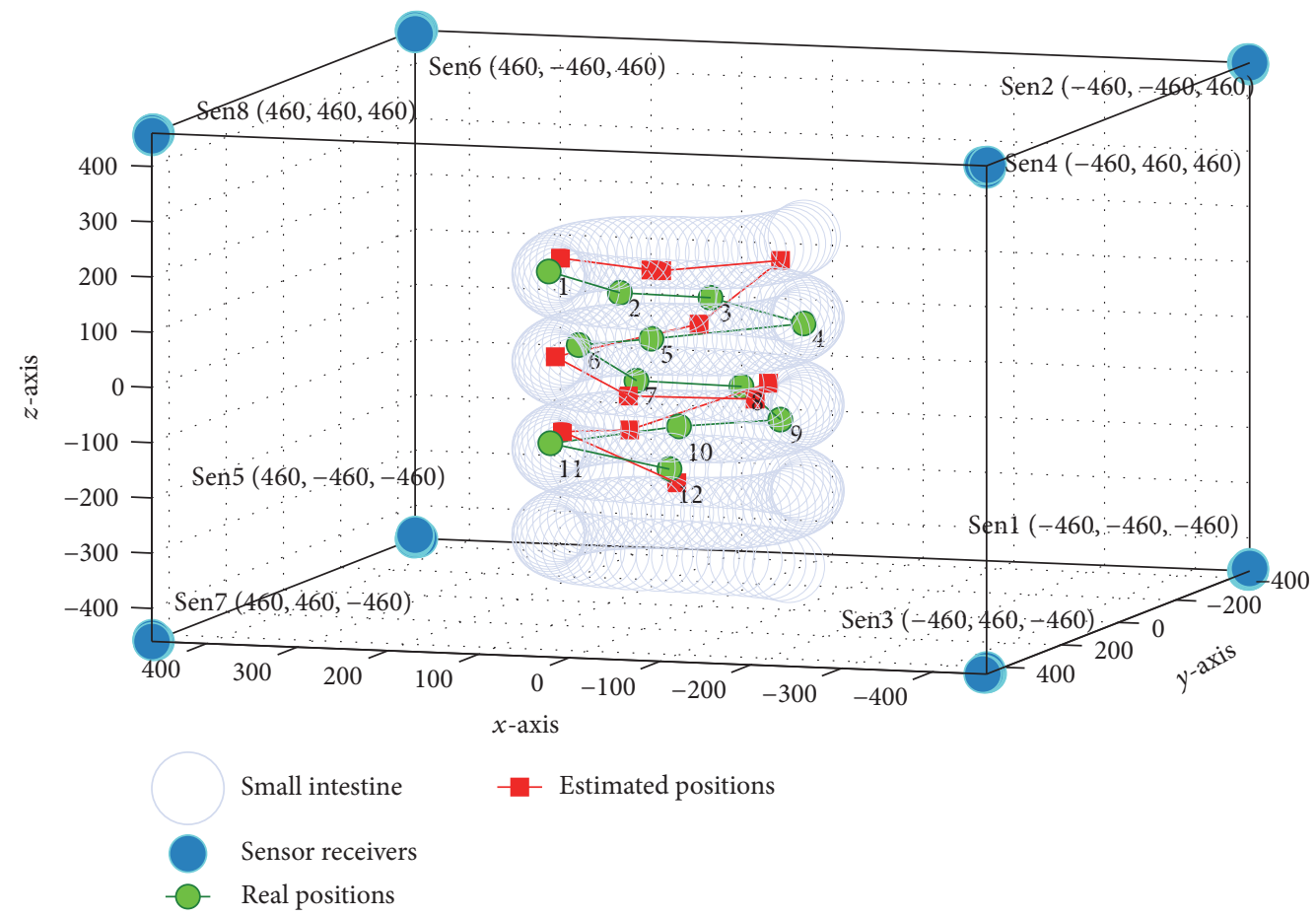

FIGURE 12: Simulation results using proposed method of scattered path loss based WCL.

distribution with $9 \mathrm{~mm}$ variance as compared to the training phase positions). We can observe that considering randomness in target positions we obtain RMSE of $13.48 \mathrm{~mm}$ accuracy using optimal coefficient where RMSE of $11.00 \mathrm{~mm}$ is achievable using the proposed suboptimal method. We also simulate our proposed algorithm considering $1-10 \%$ error in local path loss parameters and observe that our proposed suboptimal localization algorithm can obtain $11.06-13.87 \mathrm{~mm}$ RMSE accuracy. Thus it can be said that the performance of our proposed algorithm is robust to network dimension, deviation in path loss, and random variations in capsule movement and also less sensitive to local parameters error.

\section{Conclusion}

In this paper, we have proposed scattered path loss based WCL algorithm to localize the video endoscopic capsule while it travels through the small intestine. To address the randomness in path loss, we have proposed local path loss parameter dependent path loss representation to estimate the distance using randomly scattered measured path loss. The estimated distances have been used to estimate the position of the target using calibrated WCL. We have proposed a realistic suboptimal method of finding the calibration coefficient and also computed the optimal coefficient to set the benchmark of the proposed algorithm. We have developed a simulation platform to present and verify the results using different performance indices. The impacts of the number of sensors, network topology, and dimension have also been analyzed. As we have observed the proposed local parameter dependent

localization approach can reach the benchmark accuracy using randomly scattered path loss and perform equally well with changes in network dimension and random variation in target positions. We have also compared the accuracy of our proposed algorithm to other works and found improved accuracy as compared to other algorithms.

\section{Notations}

M:

$N$ :

$k$ :

$P_{T}$ :

$d(i, j):$

$d_{\max }:$

$\operatorname{PL}\left(d_{0}\right):$

$\alpha:$

$X_{\sigma}$ :

$m_{1}$ and $m_{2}$ :

$m_{1}(i, j)$ and $m_{2}(i, j)$ : Local path loss parameters of sensor $j$
Number of total positions of the target (1-2529 for our simulation system) Number of sensors used to localize the capsule which is 64 in our proposed algorithm

Number of target positions in a cluster Capsule transmitted power in $\mathrm{dB}$ Distance of the $j$ th sensor and target for $i$ th position of the target Maximum distance of the target and sensor The reference path loss for a reference distance $d_{0}$ which is less than $d(i, j)$ Path loss exponent dependent on environment Zero-mean Gaussian random variable (in $\mathrm{dB}$ ) with standard deviation $\sigma$ (in $\mathrm{dB})$ Matrices $(M \times N)$ of local path loss parameters of all sensors for target at location $i$ 


\begin{tabular}{|c|c|}
\hline$S(i, j):$ & $\begin{array}{l}\text { Scattered path loss (training phase) } \\
\text { between } j \text { th sensor and } i \text { th position of } \\
\text { the target }\end{array}$ \\
\hline $\mathrm{PL}(i, j):$ & $\begin{array}{l}\text { Measured path loss (test phase) } \\
\text { between } j \text { th sensor and } i \text { th position of } \\
\text { the target }\end{array}$ \\
\hline $\operatorname{EPL}(i, j):$ & $\begin{array}{l}\text { Adaptive LSE estimated path loss as a } \\
\text { function of distance } d_{i}\end{array}$ \\
\hline$S:$ & $\begin{array}{l}\text { Vector }(M \times 1) \text { containing the } \\
\text { scattered path loss (training phase) of } \\
2529 \text { positions }\end{array}$ \\
\hline PL: & $\begin{array}{l}\text { Vector }(M \times 1) \text { containing the } \\
\text { scattered path loss (test phase) of } 2529 \\
\text { positions }\end{array}$ \\
\hline EPL: & $\begin{array}{l}\text { Vector }(M \times 1) \text { containing the } \\
\text { adaptive LSE estimated path loss as a } \\
\text { function of distance }\end{array}$ \\
\hline$W(i, j):$ & $\begin{array}{l}\text { Weight of the } j \text { th sensor for } i \text { th } \\
\text { position of the target }\end{array}$ \\
\hline A: & $\begin{array}{l}\text { Matrix }(M \times 3) \text { containing the } \\
\text { logarithm of distance and the 1's data }\end{array}$ \\
\hline$X:$ & $\begin{array}{l}\text { Vector }(3 \times 1) \text { containing scattered } \\
\text { path loss parameters } S\left(d_{0}\right), \alpha \text { and } X_{\sigma}\end{array}$ \\
\hline$H:$ & $\begin{array}{l}\text { Matrix }(M \times 3) \text { containing the } \\
\text { logarithm of distance and 1's }\end{array}$ \\
\hline$\Theta:$ & $\begin{array}{l}\text { Matrix }(2 \times 1) \text { containing the } \\
\text { estimated path loss parameters } \\
m_{1}(i, j) \text { and } m_{2}(i, j)\end{array}$ \\
\hline$\Lambda:$ & $\begin{array}{l}\text { Matrix }(k \times 1) \text { containing the } \\
\text { scattered path loss (training phase) of } \\
k \text { positions in a cluster }\end{array}$ \\
\hline$\hbar:$ & $\begin{array}{l}\text { Matrix }(k \times 2) \text { containing the } \\
\text { logarithm of distance and l's }\end{array}$ \\
\hline$\theta:$ & $\begin{array}{l}\text { Matrix }(2 \times 1) \text { containing the } \\
\text { estimated path loss parameters for a } \\
\text { specific cluster }\end{array}$ \\
\hline$c:$ & Calibration coefficient \\
\hline$c_{\text {opt }}:$ & Optimal calibration coefficient \\
\hline$c_{\text {sopt }}:$ & Suboptimal calibration coefficient \\
\hline$B_{j}:$ & The $j$ th sensor's position \\
\hline$x_{j}, y_{j}$ and $z_{j}:$ & $j$ th sensor's $x, y$ and $z$ coordinate \\
\hline$R:$ & $\begin{array}{l}M \text { real positions of the target in } \\
\text { vector form }\end{array}$ \\
\hline$R_{\max }:$ & $\begin{array}{l}\text { Maximum range of real positions in } \\
x-y-z \text { direction }\end{array}$ \\
\hline$R_{\min }:$ & $\begin{array}{l}\text { Minimum range of real positions in } \\
x-y-z \text { direction }\end{array}$ \\
\hline$E:$ & $\begin{array}{l}M \text { estimated positions (using } \\
\text { traditional WCL) of the target in } \\
\text { vector form }\end{array}$ \\
\hline$E_{i}$ : & $\begin{array}{l}\text { Estimated position for } i \text { th location of } \\
\text { target using traditional WCL }\end{array}$ \\
\hline$P:$ & $\begin{array}{l}M \text { estimated positions (using } \\
\text { proposed WCL) of the target in vector } \\
\text { form }\end{array}$ \\
\hline$P_{i}:$ & $\begin{array}{l}\text { Estimated position for } i \text { th location of } \\
\text { target using proposed WCL }\end{array}$ \\
\hline$x_{e}, y_{e}$ and $z_{e}$ : & $\begin{array}{l}x, y \text { and } z \text { coordinate of estimated } \\
\text { position }\end{array}$ \\
\hline
\end{tabular}

$x_{r}, y_{r}$ and $z_{r}: x, y$ and $z$ coordinate of real position

$\mathrm{LE}_{i}$ : $\quad$ Localization error for $i$ th position of target

ALE: Average localization error

RMSE: $\quad$ Root mean square error

NRMSE: Normalized root mean square error

STD: $\quad$ Standard deviation of localization error.

\section{Conflicts of Interest}

The authors declare that there are no conflicts of interest regarding the publication of this paper.

\section{References}

[1] Z. Farid, R. Nordin, and M. Ismail, "Recent advances in wireless indoor localization techniques and system," Journal of Computer Networks and Communications, vol. 2013, Article ID 185138, 12 pages, 2013.

[2] W. Murphy and W. Hereman, Determination of a Position in Three Dimensions Using Trilateration and Approximate Distances, vol. 7, Department of Mathematical and Computer Sciences, Colorado School of Mines, Colorado, Colo, USA, 1995.

[3] B. Hofmann-Wellenhof, H. Lichtenegger, and J. Collins, GPSGlobal Positioning System: Theory And Practice, vol. 1, Springer, Wien, Austria, 1997.

[4] B. Favre-Bulle, J. Prenninger, and C. Eitzinger, "Efficient tracking of 3 D-robot positions by dynamic triangulation," in Proceedings of the IEEE Instrumentation and Measurement Technology Conference, vol. 1, pp. 446-449, 1998.

[5] N. Bulusu, J. Heidemann, and D. Estrin, "GPS-less low-cost outdoor localization for very small devices," IEEE Personal Communications, vol. 7, no. 5, pp. 28-34, 2000.

[6] J. Blumenthal, R. Grossmann, F. Golatowski, and D. Timmermann, "Weighted centroid localization in Zigbee-based sensor networks," in Proceedings of the IEEE International Symposium on Intelligent Signal Processing (WISP '07), pp. 1-6, Alcalá de Henares, Spain, October 2007.

[7] K. Pahlavan, G. Bao, Y. Ye et al., "RF localization for wireless video capsule endoscopy," International Journal of Wireless Information Networks, vol. 19, no. 4, pp. 326-340, 2012.

[8] C. Hu, M. Li, S. Song, W. Yang, R. Zhang, and M. Q.-H. Meng, "A cubic 3-axis magnetic sensor array for wirelessly tracking magnet position and orientation," IEEE Sensors Journal, vol. 10, no. 5, pp. 903-913, 2010.

[9] J. Hou, Y. Zhu, L. Zhang et al., "Design and implementation of a high resolution localization system for in-vivo capsule endoscopy," in Proceedings of the 8th IEEE International Symposium on Dependable, Autonomic and Secure Computing (DASC '09), pp. 209-214, December 2009.

[10] K. Arshak and F. Adepoju, "Adaptive linearized methods for tracking a moving telemetry capsule," in Proceedings of the IEEE International Symposium on Industrial Electronics (ISIE '07), pp. 2703-2708, June 2007.

[11] Y. Wang, R. Fu, Y. Ye, U. Khan, and K. Pahlavan, "Performance bounds for RF positioning of endoscopy camera capsules," in Proceedings of the IEEE Topical Conference on Biomedical Wireless Technologies, Networks, and Sensing Systems (BioWireleSS '11), pp. 71-74, Phoenix, Ariz, USA, January 2011. 
[12] Y. Ye, P. Swar, K. Pahlavan, and K. Ghaboosi, "Accuracy of RSS-based RF localization in multi-capsule endoscopy," International Journal of Wireless Information Networks, vol. 19, no. 3, pp. 229-238, 2012.

[13] D. Anzai, S. Aoyama, and J. Wang, "Impact of propagation characteristics on RSSI-based localization for $400 \mathrm{MHz}$ MICS band implant body area networks," in Proceedings of the IEEE International Symposium on Medical Information and Communication Technology, pp. 1-4, March 2012.

[14] M. Pourhomayoun, M. Fowler, and Z. Jin, "A novel method for medical implant in-body localization," in Proceedings of the 34th Annual International Conference of the IEEE Engineering in Medicine and Biology Society, EMBS 2012, pp. 5757-5760, September 2012.

[15] R. Chandra, A. J. Johansson, and F. Tufvesson, "Localization of an RF source inside the human body for wireless capsule endoscopy," in Proceedings of the International Conference on Body Area Networks, pp. 48-54, Institute for Computer Sciences, Social-Informatics and Telecommunications Engineering, Boston, Mass, USA, October 2013.

[16] S. T. Goh, S. A. R. Zekavat, and K. Pahlavan, "DOA-based endoscopy capsule localization and orientation estimation via unscented kalman filter," IEEE Sensors Journal, vol. 14, no. 11, pp. 3819-3829, 2014.

[17] G. Bao, K. Pahlavan, and L. Mi, "Hybrid localization of microrobotic endoscopic capsule inside small intestine by data fusion of vision and RF sensors," IEEE Sensors Journal, vol. 15, no. 5, pp. 2669-2678, 2015.

[18] U. Hany and K. A. Wahid, "An adaptive linearized method for localizing video endoscopic capsule using weighted Centroid algorithm," International Journal of Distributed Sensor Networks, vol. 11, no. 3, p. 342428, 2015.

[19] U. Hany, L. Akter, and F. Hossain, "Degree-based wcl for video endoscopic capsule localization," IEEE Sensors Journal, vol. 17, no. 9, pp. 2904-2916, 2017.

[20] U. Hany and L. Akter, "Non-parametric approach of video capsule endoscope localization using suboptimal method of position bounded CWCL," IEEE Sensors Journal, vol. 17, no. 20, pp. 6806-6815, 2017.

[21] K. Sayrafian-Pour, W.-B. Yang, J. Hagedorn, J. Terrill, and K. Y. Yazdandoost, "A statistical path loss model for medical implant communication channels," in Proceedings of the IEEE 20th Personal, Indoor and Mobile Radio Communications Symposium (PIMRC '09), pp. 2995-2999, Tokyo, Japan, September 2009.

[22] L. Wang, L. Liu, C. Hu, and M. Q. Meng, "A novel RF-based propagation model with tissue absorption for location of the GI tract," IEEE Annual International Conference on Engineering in Medicine and Biology Society, pp. 654-657, 2010.

[23] F. Ileri and M. Akar, "RSSI based position estimation in zigbee sensor networks," WSEAS Recent Advances in Circuits, Systems, Signal Processing and Communications, pp. 62-73, 2014.

[24] Z.-M. Wang and Y. Zheng, "The study of the weighted centroid localization algorithm based on RSSI," in Proceedings of the 2014 International Conference on Wireless Communication and Sensor Network, WCSN 2014, pp. 276-279, Wuhan, China, December 2014.

[25] J. Wang, P. Urriza, Y. Han, and D. Cabric, "Weighted centroid localization algorithm: theoretical analysis and distributed implementation," IEEE Transactions on Wireless Communications, vol. 10, no. 10, pp. 3403-3413, 2011.

[26] Z. Zeng, J. Gao, and J. Wang, "Corrected range weighted centroid localization algorithm based on RSSI for WSN," in
Proceedings of the 2011, International Conference on Informatics, Cybernetics, and Computer Engineering (ICCE2011), vol. 111, pp. 453-460, Springer, Melbourne, Australia, 2011.

[27] A. Fink and H. Beikirch, "Refinement of weighted centroid localization using a regular infrastructure topology," in Proceedings of the 5th International Conference on Indoor Positioning and Indoor Navigation, IPIN 2014, pp. 1-10, Busan, South Korea, October 2014

[28] J. Zhao, Q. Zhao, Z. Li, and Y. Liu, "An improved weighted centroid localization algorithm based on difference of estimated distances for wireless sensor networks," Telecommunication Systems, vol. 53, no. 1, pp. 25-31, 2013.

[29] D. Y. Zou, H. Lv, D. L. Zheng, and T. F. Han, "An improved weighted centroid localization algorithm in WSN," Applied Mechanics and Materials, vol. 513-517, pp. 3496-3499, 2014.

[30] Q. Dong and X. Xu, "A novel weighted centroid localization algorithm based on RSSI for an outdoor environment," Journal of Communications, vol. 9, no. 3, pp. 279-285, 2014.

[31] B. Zhang, J. Zhu, P. Gao, and J. Xu, "The improvement of RSSI for mine personnel positioning," in Proceedings of the 2015 International Conference on Intelligent Systems Research and Mechatronics Engineering, Zhengzhou, China, April 2015.

[32] T. S. Rappaport, Wireless Communications: Principles and Practice, vol. 2 of New Jersey, NJ, USA, Prentice Hall, 2015.

[33] S. M. Kay, Fundamentals of Statistical Signal Processing: Estimation Theory, vol. 1, Prentice Hall, 1993. 


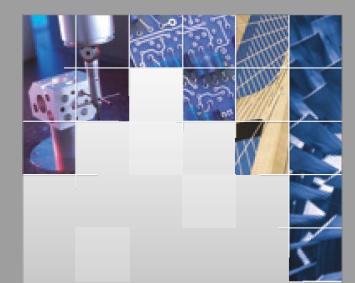

\section{Enfincering}
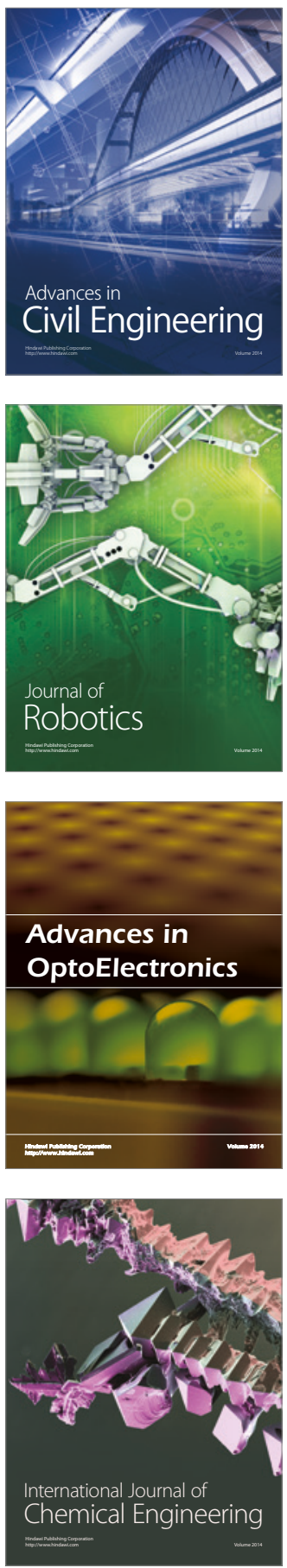

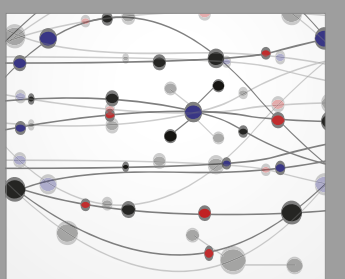

The Scientific World Journal

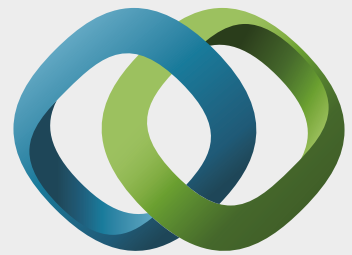

\section{Hindawi}

Submit your manuscripts at

https://www.hindawi.com
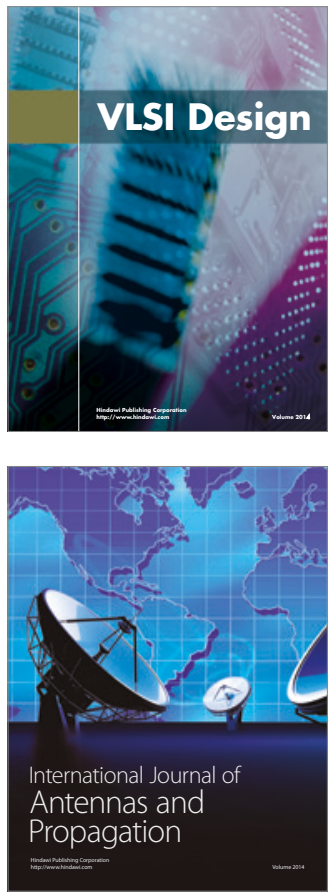

\section{Rotating}

Machinery
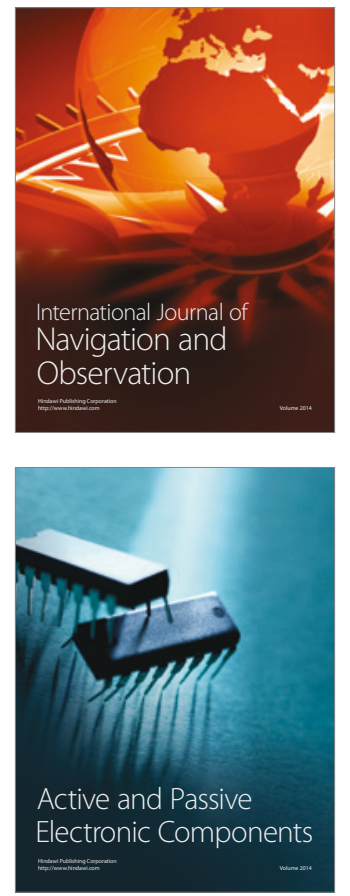
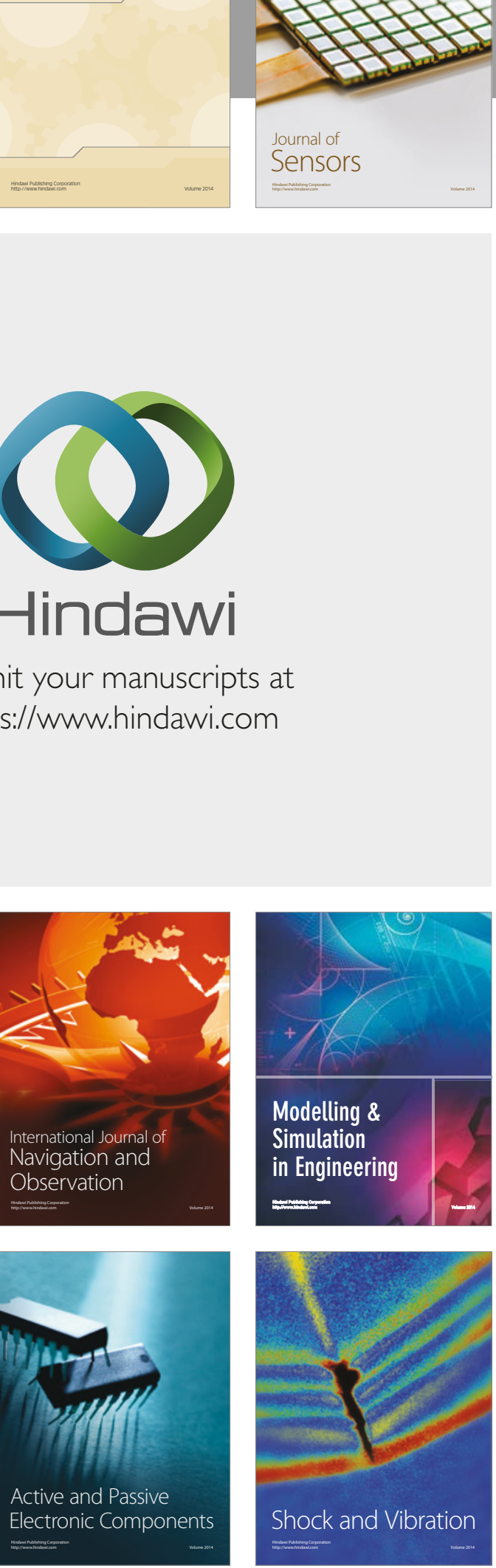
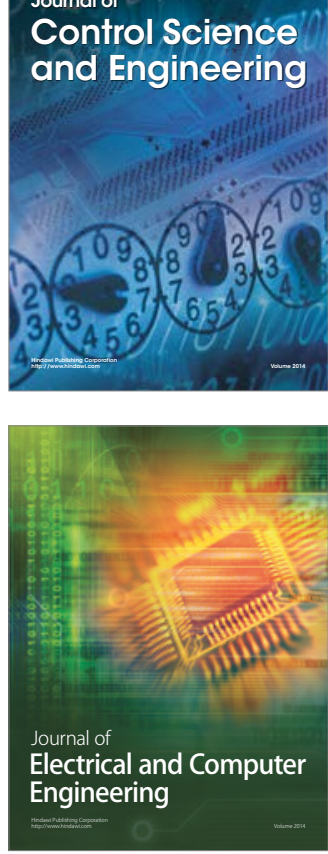

Distributed

Journal of

Control Science

and Engineering
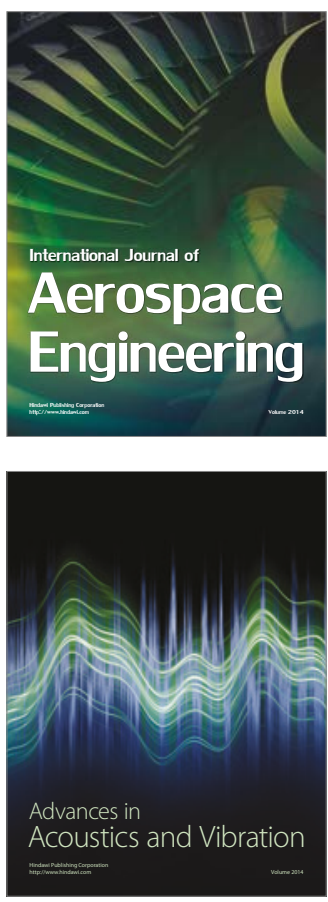

Sensor Networks 